\title{
Linear stability studies for a quasi-axisymmetric stellarator configuration including effects of parallel viscosity, plasma flow, and resistive walls
}

\author{
E. Strumberger and S. Günter \\ Max Planck Institute for Plasma Physics, Boltzmannstr. 2. \\ 85748 Garching, Germany
}

August 8, 2019

\begin{abstract}
We present linear stability studies performed with the newly developed CASTOR3D code. This code is a very flexible and versatilely applicable numerical tool to study the stability properties of 3D equilibria. Ideal external kink modes of a quasi-axisymmetric (QA) equilibrium are investigated taking into account effects of parallel viscosity, plasma flow, and resistive walls. The results are compared with those obtained for an axisymmetric approximation of this equilibrium. Two major differences in the stability properties are found: (i) The QA equilibrium is vertical stable, while the axisymmetric equilibrium is vertical unstable. (ii) A mode of the QA equilibrium only oscillates, when the plasma rotates with a minimum rotation frequency, or faster. This mininum rotation frequency depends on the $n^{*}$-type of mode (with $n^{*}$ being the dominant toroidal Fourier harmonic contributing to the mode). Resistive wall structures in combination with plasma flow, however, lead to resistive wall modes and/or plasma modes for both types of equilibria.
\end{abstract}

Keywords: linear stability, (quasi)-axisymmetric equilibria, external kink modes, plasma rotation, resistive walls

email address of the corresponding author: erika.strumberger@ipp.mpg.de (E. Strumberger)

(The figures appear in colour only in the electronic version) 


\section{Introduction}

Three-dimensional tokamak and stellarator equilibria are in the focus of present fusion research. While stellarators are characterized by a complex 3D magnetic field topology, 3D tokamaks are devices with weakly broken axisymmetry. Reasons for the asymmetry of tokamak configurations are e.g. three-dimensional resistive wall structures which can reduce the growth rates of external modes [1, 2, 3], and magnetic perturbation fields [4]. The latter are applied to mitigate, or even to suppress edge localized modes (ELMs) [5, 6]. In quasi-axisymmetric (QA) configurations [7] the rotational transform is partly produced by coil currents, while the magnetic field strength is approximately independent from the toroidal Boozer coordinate [8]. That is, these configurations have common properties with tokamaks and stellarators. The question, whether a QA configuration can be found that unites the positive properties of tokamaks and stellarators, is currently investigated [9]. The design of 3D fusion devices, as well as the analysis and interpretation of the corresponding plasma discharges require appropriate numerical tools that are able to handle their complex geometry.

There are several 2D reduced and full Magneto HydroDynamic (MHD), linear stability codes which take into account important physical effects, such as, resistivity, viscosity, and plasma flow, (e.g. MARS [10], CASTOR_FLOW [11], MISHKA-F [12]), drift-kinetic effects on resistive wall modes (MARS-K [13]), and the stabilizing effects of thick 3D walls (CarMa [14]). However, in their present forms all these codes can only be applied to 2D axisymmetric equilibria. There are also 3D linear stability codes (e.g. CAS3D [15], TERPSICHORE [16], etc.), but these are ideal stability codes relying on the energy principle. To our knowledge, the newly developed, linear stability CASTOR3D code [17] is presently the only linear stability code that works for $2 \mathrm{D}$ and $3 \mathrm{D}$ equilibria, and takes plasma resistivity, viscosity, and torodial flow into account.

The CASTOR3D code is a hybrid version of the linear stability CASTOR_3DW code [18], and the resistive wall mode STARWALL code [3], which uses the thin wall approximation. Both code parts have been modified and extended considerably. Their general 3D formulation allows to use various kinds of flux coordinates. There is no limitation to ideal wall structures or resistive time scales, because the extended eigenvalue problem describes simultaneously the plasma perturbation and the corresponding currents in the external conducting structures. Depending on the complexity of the conducting structures, they are either discretized by a spectral method, or by triangular finite elements where the resistivity in each triangle may be different.

Several code extensions and improvements have been made in comparison to the preceding 
code version described in Ref. [17]. In addition to plasma resistivity, the current code version includes the effects of parallel viscosity, and plasma flow in the direction of (quasi-)symmetry. The coupling of the linear MHD and the vacuum equations via the perturbed magnetic vector potential allows the solution of an extendend eigenvalue problem including the effects of plasma resistivity, parallel viscosity, and plasma flow. This non-hermitian eigenvalue problem is solved by using the parallelized SLEPc-Krylov-Schur solver [19].

During the optimization process of QA stellarator equilibria [9] also a highly compact, but rather unstable configuration was found. Because of its relatively simple geometry and its instability, this QA equilibrium is studied in the following. It provides an excellent test case to demonstrate the large variety of possible applications of the CASTOR3D code. In order to illustrate similarities and differences of the stability properties of axisymmetric and quasi-axisymmetric configurations, also an axisymmetric approximation of this stellarator configuration is investigated.

The paper is organized as follows. In section 2 necessary extensions and modifications of the eigenvalue problem are described. The properties of the considered equilibria are summarized in section 3. In section 4 linear stability studies are presented for ideal, external kink modes (section 4.1) taking into account the effects of parallel viscosity (section 4.2), plasma flow (section 4.3), and resistive wall structures (section 4.4). Code properties and obtained results are summarized in section 5, and in the appendix the numerical accuracy of the computations is discussed.

\section{Extensions and modifications of the extended eigenvalue problem}

The numerical methods used in the CASTOR3D code are described in detail in [17] and [3]. However, the linearized MHD equations used in [17] do not take into account plasma flow and parallel viscosity, and the extended eigenvalue problem is only solved for an ideal plasma. Using the gauge $\vec{B}=\nabla \times \vec{A}, \vec{E}=-\lambda \vec{A}$, with $\vec{B}$ and $\vec{E}$ being the perturbations of magnetic and electric fields [20], the single-fluid, resistive MHD equations including flow and viscosity [11] read as follows:

$$
\begin{gathered}
\lambda \rho=-\vec{V}_{0} \cdot \nabla \rho-\rho \nabla \cdot \vec{V}_{0}-\vec{v} \cdot \nabla \rho_{0}-\rho_{0} \nabla \cdot \vec{v} \\
\lambda \rho_{0} \vec{v}=-\rho\left(\vec{V}_{0} \cdot \nabla\right) \vec{V}_{0}-\rho_{0}\left(\left(\vec{V}_{0} \cdot \nabla\right) \vec{v}+(\vec{v} \cdot \nabla) \vec{V}_{0}\right)-\nabla\left(\rho_{0} T+\rho T_{0}\right) / m
\end{gathered}
$$




$$
\begin{gathered}
-\nabla \cdot \boldsymbol{\pi}_{\|}+\left(\left(\nabla \times \vec{B}_{0}\right) \times(\nabla \times \vec{A})+(\nabla \times(\nabla \times A)) \times \vec{B}_{0}\right) / \mu_{0}, \\
\lambda T=-\vec{V}_{0} \cdot \nabla T-(\Gamma-1) T \nabla \cdot \vec{V}_{0}-\vec{v} \cdot \nabla T_{0}-(\Gamma-1) T_{0} \nabla \cdot \vec{v}, \\
\lambda \vec{A}=\vec{V}_{0} \times(\nabla \times \vec{A})+\vec{v} \times \vec{B}_{0}-\frac{\eta}{\mu_{0}} \nabla \times(\nabla \times \vec{A}) .
\end{gathered}
$$

Here, $\rho, \vec{v}, T$, and $\vec{A}$ describe the perturbations of density, velocity, temperature and magnetic vector potential, respectively. The time dependence of the perturbed quantities is taken to be $\sim e^{\lambda t}$, with $\lambda=\gamma+i \omega$. The real part of $\lambda$ denotes the growh rate, and $\omega$ describes the oscillation frequency of the mode. The quantities $\rho_{0}$ and $T_{0}$ represent the unperturbed density and temperature, while $m, \mu_{0}, \eta$, and $\Gamma(\Gamma=5 / 3)$ indicate particle mass, vacuum permability, plasma resistivity, and compressibility.

Symmetric and quasi-symmetric plasma configurations can rotate almost freely in the direction of (quasi-)symmetry [21]. Therefore, we use the general ansatz for the plasma velocity

$$
\vec{V}_{0}=\frac{1}{2 \pi} \Omega(s) \vec{r}_{v} .
$$

Here, $s, v, u$ are the curvilinear coordinates with the radial coordinate, $s$, being defined as the square root of the normalized toroidal flux, $v$ being the toroidal, and $u$ being the poloidal coordinate. The rotational frequency, $\Omega$, is only a function of $s$. The toroidal coordinate $v$ corresponds to the direction of symmetry for (i) axisymmetric equilibria using axisymmetric straight field line coordinates $[22,20]$, (ii) axisymmetric and almost axisymmetric 3D tokamak configurations using the flux coordinates of the NEMEC code [23, 24], and (iii) QA configurations using Boozer coordinates [8]. That is, this general ansatz of the plasma flow holds for various types of symmetries provided the proper coordinates are chosen.

Toroidal flow in combination with dissipation in the plasma may open a stability window within a certain distance range of an external wall $[25,10]$. In a first step, we indroduce a simple form of the parallel viscosity

$$
-\nabla \cdot \boldsymbol{\pi}_{\|} \approx \mu_{\|} \Delta_{\|} \vec{v}
$$

with the velocity perturbation, $\vec{v}$, dynamic viscosity coefficient, $\mu_{\|}$, and the Laplace operator in parallel direction, $\Delta_{\|}$. The latter is defined by

$$
\Delta_{\|}=\vec{b}(\vec{b} \cdot \nabla)(\vec{b}(\vec{b} \cdot \nabla))
$$

with the unit vector, $\vec{b}=\frac{\vec{B}_{0}}{B_{0}}$, pointing in direction of the equilibrium magnetic field, $\vec{B}_{0}=$ $B^{v} \vec{r}_{, v}+B^{u} \vec{r}_{,_{u}}$. 
As described in detail in [17] and [3], the vacuum equations are derived from a Lagrangian using a variational method. There, the solution for the perturbation of the vacuum vector potential can be generated by surface currents on the plasma-vacuum boundary, and on the resistive and ideal wall structures. This Lagrangian contains the boundary term $\int_{S_{p}} d f_{p} \vec{K}_{p} \cdot \vec{A}^{v a c}$ with $\vec{K}_{p}$ being the divergence-free surface current density at the plasma boundary, $S_{p}$, and $\overrightarrow{A^{v a c}}$ being the perturbation of vector potential at this boundary.

The tangential component of the electric field perturbation is continuous at the plasma boundary

$$
\overrightarrow{n_{p}} \times \vec{E}^{v a c}=\overrightarrow{n_{p}} \times \vec{E}
$$

with $n_{p}$ being the normal vector of the plasma surface in outward direction. Using $\vec{E}=-\frac{\partial \vec{A}}{\partial t}=$ $-\lambda \vec{A}$ together with Ohm's law (4), the boundary condition (8) reads

$$
\overrightarrow{n_{p}} \times \vec{A}^{v a c}=-\frac{1}{\lambda}\left(\left(\overrightarrow{n_{p}} \cdot \vec{B}\right) \vec{V}_{0}+\left(\vec{n}_{p} \cdot \vec{v}\right) \vec{B}_{0}+\frac{\eta}{\mu_{0}} \vec{n}_{p} \times(\nabla \times \vec{B})\right)=\overrightarrow{n_{p}} \times \vec{A}
$$

Equation (9) reduces to the boundary condition used in [17] in case of an ideal plasma without flow. There, the vacuum equations are coupled with the MHD equations via the radial velocity perturbation $\vec{n}_{p} \cdot \vec{v}$ at the plasma boundary $\left(\overrightarrow{n_{p}} \times \overrightarrow{A^{v a c}}=-\frac{1}{\lambda}\left(\left(\overrightarrow{n_{p}} \cdot \vec{v}\right) \overrightarrow{B_{0}}\right)\right.$. However, (9) offers also a much simplier way, namely

$$
\overrightarrow{n_{p}} \times \overrightarrow{A^{v a c}}=\overrightarrow{n_{p}} \times \vec{A}
$$

Using this relation and performing the transformations

$$
\overrightarrow{n_{p}} \cdot\left(\vec{K}_{p} \times\left(\overrightarrow{n_{p}} \times \overrightarrow{A^{v a c}}\right)=\overrightarrow{n_{p}} \cdot\left(\vec{K}_{p} \times\left(\overrightarrow{n_{p}} \times \vec{A}\right)\right.\right.
$$

yields

$$
\vec{K}_{p} \cdot \vec{A}^{v a c}=\vec{K}_{p} \cdot \vec{A}
$$

That is, the vacuum equations can also be coupled with the MHD equations via the vector potential perturbation of the plasma, $\vec{A}$. This coupling is independent of the explicit form of Ohm's law (4). 


\section{Equilibria}

The considered QA equilibrium is characterized by geometrical and physical quantities listed in Table 1. Based on this equilibrium, an axisymmetric equilibrium has been computed by leaving pressure, rotational transform and plasma beta unchanged, and defining the plasma boundary by the $n=0$ Fourier harmonics of the QA boundary. As shown in Table 1, the axisymmetric equilibrium has similar properties as the quasi-axisymmetric one. However, the toroidal plasma current of the axisymmetric equilibrium is larger since most of the poloidal field has to be created by this current, and not by complicated shaped external coils as in the QA case.

Table 1: Properties of the quasi-axisymmetric and axisymmetric equilibria.

\begin{tabular}{llrrr}
\hline \hline quantity & & unit & quasi-axisym. & axisym. \\
\hline aspect ratio: & $A$ & & 4.113 & 4.038 \\
major radius: & $R_{0}$ & {$[\mathrm{~m}]$} & 12.290 & 12.069 \\
minor radius: & $a_{0}$ & {$[\mathrm{~m}]$} & 2.988 & 2.923 \\
min. inboard radius: & $R_{i n}^{\min }$ & {$[\mathrm{m}]$} & 7.860 & 9.694 \\
max. outboard radius: & $R_{\text {out }}^{\max }$ & {$[\mathrm{m}]$} & 14.995 & 14.444 \\
plasma volume: & $V$ & {$\left[\mathrm{~m}^{3}\right]$} & 1900.006 & 2035.614 \\
rot. transform (axis): & $\iota_{a}$ & & 0.095 & 0.095 \\
rot. transform (boundary): & $\iota_{b}$ & & 0.557 & 0.557 \\
toroidal plasma current: & $I_{\text {tor }}$ & {$[\mathrm{MA}]$} & -8.890 & -12.402 \\
poloidal plasma current: & $J_{p o l}$ & {$[\mathrm{MA}]$} & -3.700 & -1.666 \\
total toroidal flux: & $\Phi_{\text {tot }}$ & {$[\mathrm{Wb}]$} & 125.256 & 137.00 \\
total poloidal flux: & $\Psi_{\text {tot }}$ & {$[\mathrm{Wb}]$} & 67.327 & 73.639 \\
pressure (axis): & $p_{a}$ & {$[\mathrm{kPa}]$} & 574.060 & 574.060 \\
magnetic field strength (axis): & $B_{a}$ & {$[\mathrm{~T}]$} & 4.749 & 4.728 \\
plasma beta: & $<\beta>$ & {$[\%]$} & 2.949 & 2.929 \\
\hline \hline
\end{tabular}

Figure 1 shows three cross-sections of the QA equilibrium at the toroidal angles $\varphi=0,45$ and $90^{\circ}$ in comparison with the cross-section of the axisymmetric approximation. The profiles of pressure, rotational transform and toroidal current are presented in Fig. 2. Since the rotational transform, $\iota$, is greater 0.5 (safety factor $q<2$ ) at the plasma boundary, these equilibria are highly unstable with respect to ideal, external kink modes. Therefore, these equilibria are excellent test cases for the CASTOR3D code. 

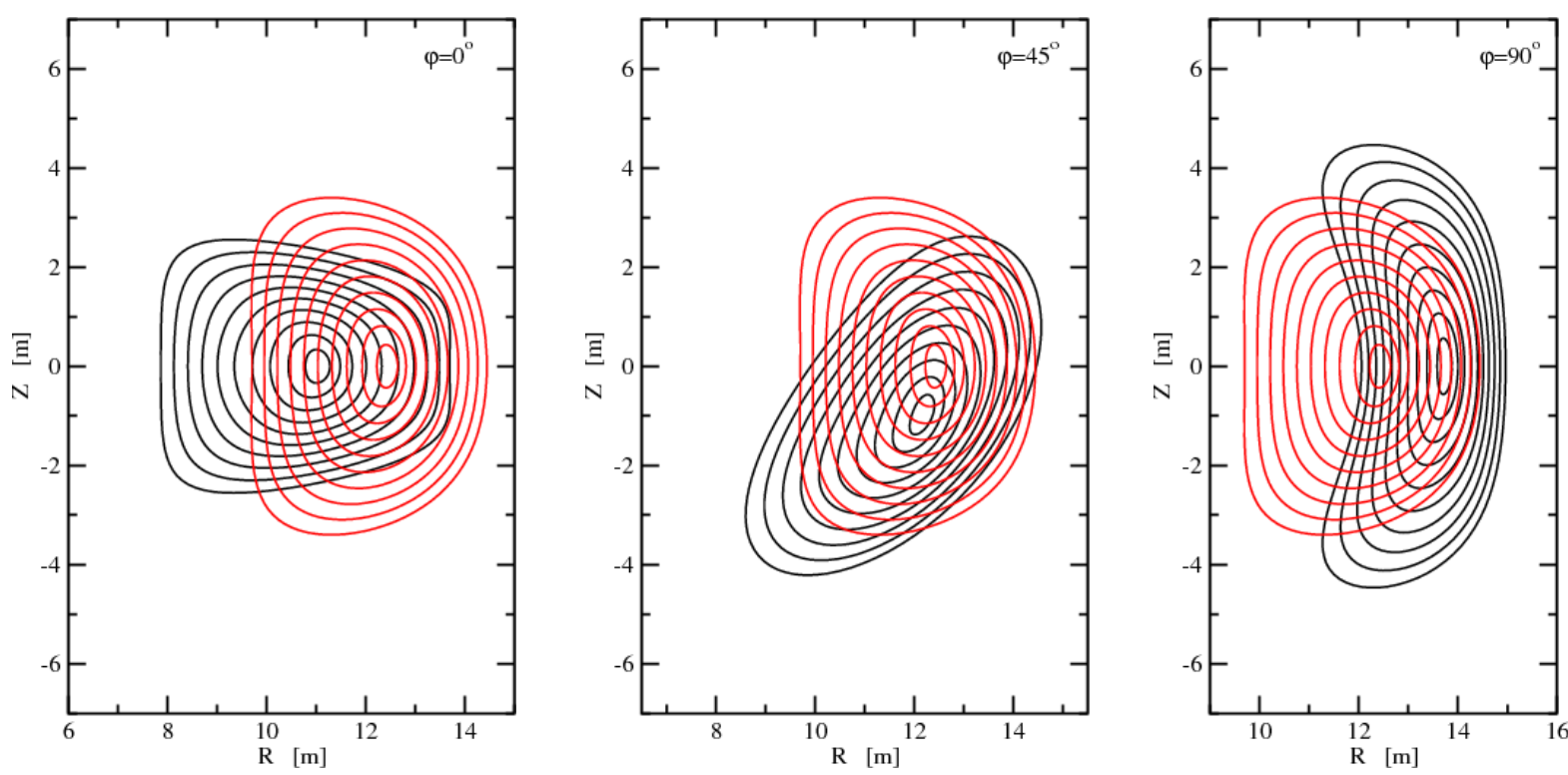

Fig. 1: Cross-sections of the $Q A$ equilibrium (black) at the toroidal angles $\varphi=0,45$, and $90^{\circ}$ in comparison with its axisymmetric approximation (red).

(a)

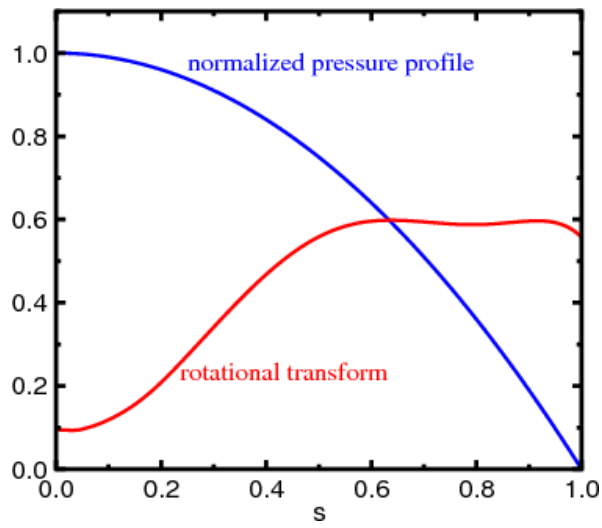

(b)

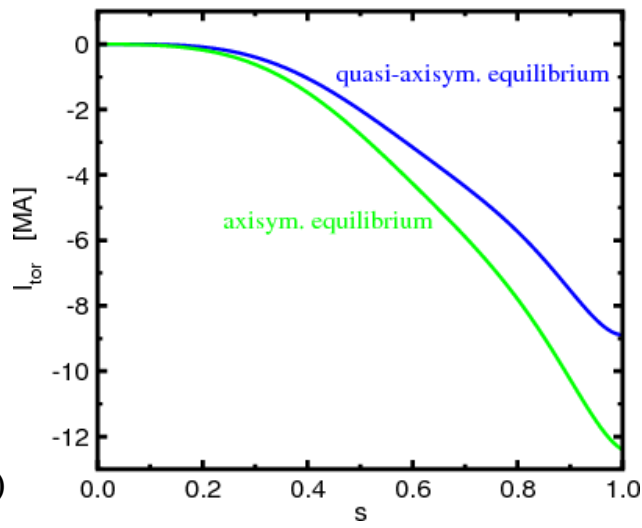

Fig. 2 (a) Rotational transform and normalized pressure profiles, which are identical for the quasi-axisymmetric and the axisymmetric configurations, and (b) toroidal current profiles.

Quasi-axisymmetry means that in Boozer coordinates the magnetic field strength is independent of the toroidal coordinate. However, this symmetry is only fulfilled approximately. Due to the design of the considered equilibrium [9], the deviation from the quasi-axisymmetry is smallest around $S \approx 0.4-0.5$ ( $S=s^{2}$ being the normalized toroidal flux), while it increases in direction to the magnetic axis and to the plasma boundary. Figure 3 shows the magnetic field strength at the plasma boundary for the QA equilibrium and the axisymmetric approximation of this equilibrium. 

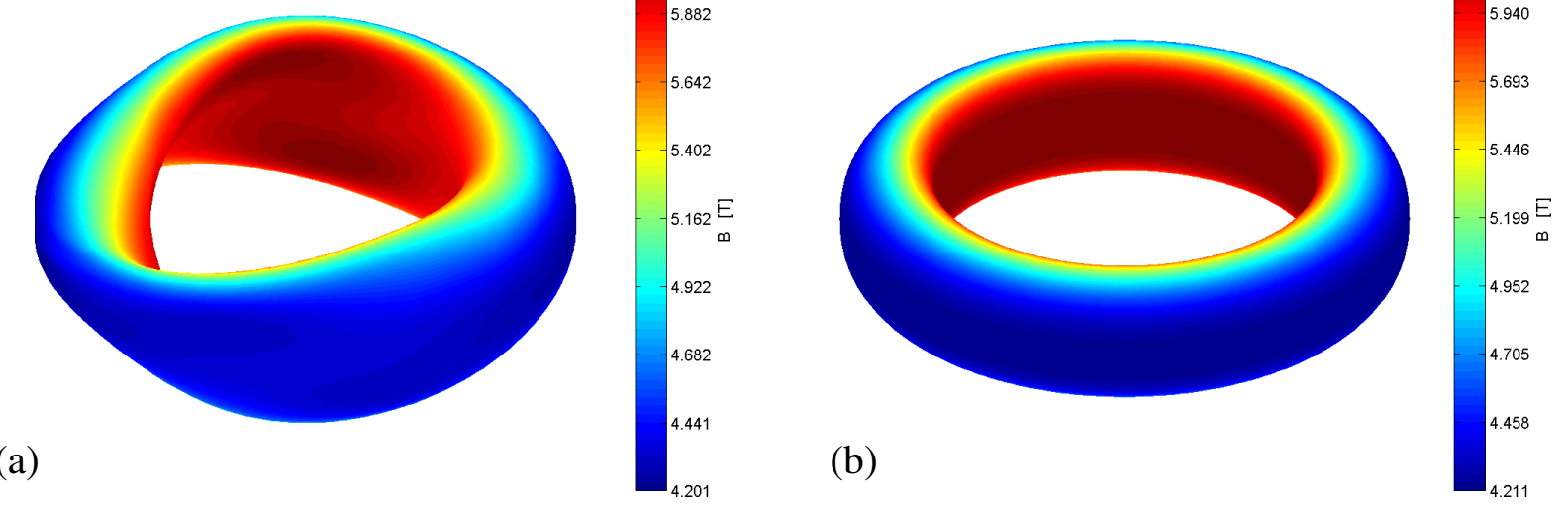

Fig. 3 Magnetic field strength at the plasma boundary for (a) the QA equilibrium, and (b) the axisymmetric approximation.

\section{Linear stability studies}

\subsection{Ideal external kink modes}

The considered QA stellarator configuration and its axisymmetric approximation are unstable with respect to various ideal, external kink modes. Solving the non-hermitian eigenvalue problem for a sub-group of eigenvalues by using the parallelized SLEPc Krylov-Schur Solver [19], sets of unstable eigenvalues are obtained. The eigenmodes are identified by viewing the corresponding eigenfunction Fourier spectra of these eigenvalues. For this purpose, we use the Fourier spectrum of the radial velocity perturbation, because it corresponds to the normal displacement. While the modes of axisymmetric equilibria are characterized by their toroidal mode number $n$, for 3D equilibria several $n$-harmonics couple together and contribute to the Fourier spectrum of the perturbation. In the latter case, we use the dominant $n$-harmonic of the Fourier spectrum, which we call $n^{*}$, to characterize these modes and their eigenvalues. This mode labeling is very useful and physically justified, as we will show below. It is $n^{*}=n$ for the modes of the axisymmetric equilibrium. In the following, we will use the lable $n^{*}$ for the modes of the 2D and 3D equilibria. More details on the mode labeling, the used sets of toroidal and poloidal Fourier harmonics, and the numerical accuracy of the results are given in the appendix. 


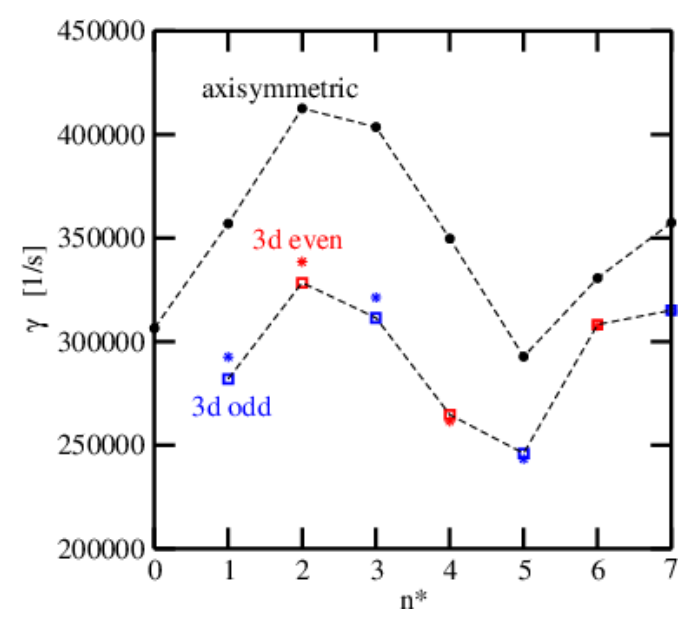

Fig. 4 Growth rates of the ideal, external kink modes as function of $n^{*}$ for the axisymmetric (black), and the QA equilibrium (blue: odd mode family, red: even mode family). Stars and squares mark the non-degenerated eigenvalues of the two orthogonal solutions of the QA case.

The growth rates as function of $n^{*}$ are shown for the axisymmetric and the quasi-axisymmetric equilibrium in Fig. 4. Because of the two-periodic QA stellarator configuration, there exist two mode families, that is, an odd $(n=1,3,5, \ldots)$ and an even $(n=0,2,4, \ldots)$ one. Only the harmonics of a mode family couple together. In contrast to the axisymmetric equilibrium, the eigenvalues of the two orthogonal solutions of each mode of the QA equilibrium are not degenerated. The differences between these two eigenvalues decrease with growing $n^{*}$, because the larger $n^{*}$, the higher the $m$ of the leading Fourier harmonic, and the more similar are the mode structures, as illustrated in Figs 5,6 and 7. Although, the quasi-axisymmetric equilibrium and its axisymmetric approximation are also unstable with respect to higher $n^{*}$-modes, we restrict our studies to $n^{*} \leq 7$. The influence of the 3D geometry is largest for low- $n^{*}$ modes, and the computational effort grows with increasing $n^{*}$.

In Figs 5, 6, and 7 the real parts of the complex and conjugate-complex Fourier harmonics, and the corresponding mode structures of the radial velocity perturbation are shown for the $n^{*}=2,3$, and 7 external kink modes. Due to the stellarator symmetry of the quasi-axisymmetric configuration, the eigenfunctions are sine- and cosine-type functions, respectively. While the labeling of modes presented in Fig. 5 and Fig. 7 by $n^{*}=2$ and $n^{*}=7$ is unambiguous (the dominant $n$-harmonics are $n=2$ and $n=7$, respectively), this is not so easy to see for the $n^{*}=3$ mode illustrated in Fig. 6 . As shown in the upper plot of Fig. 6b, the contributions of the $n=7$ harmonics are larger than the $n=3$ ones in case of the cosine-type mode. However, the eigenvalues of the sine- and cosine-type modes are very similar. There exists no further cosine-type mode with large $n=3$ contributions, and, last but not least, there is another mode with unambiguous $n^{*}=7$ character (see Fig. 7). The eigenvalue of that cosine-type mode fits very well to the eigenvalue of the $n^{*}=7$, sine-type mode, and the mode structures are also very similar. 

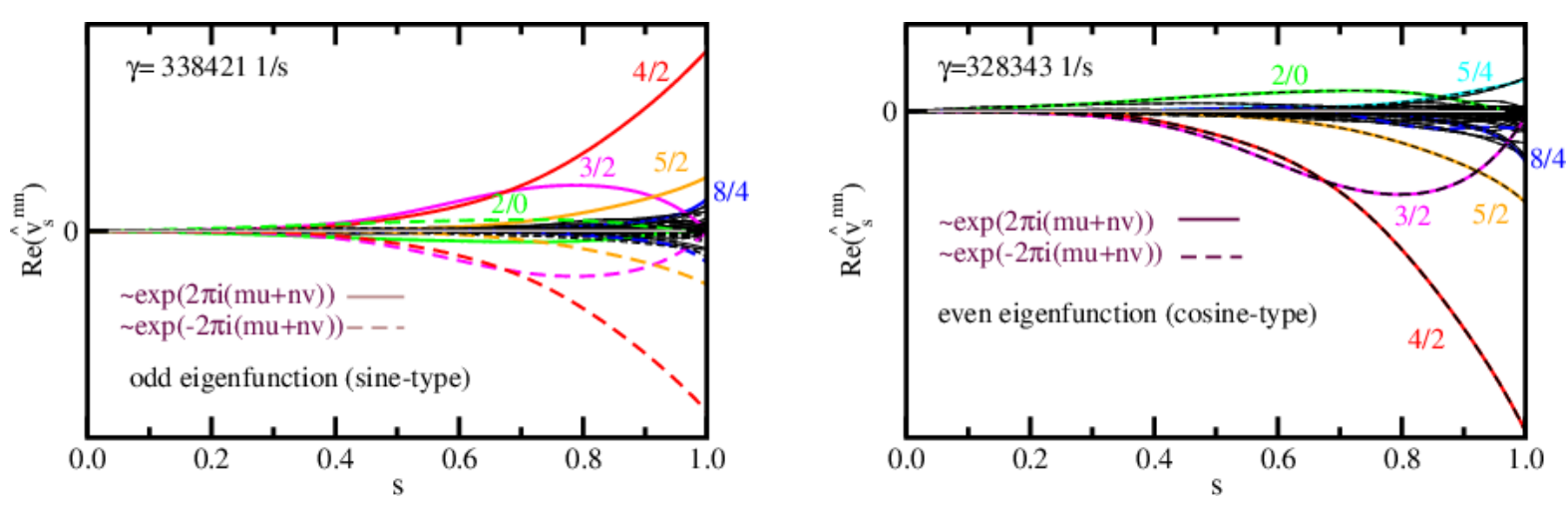

(a)

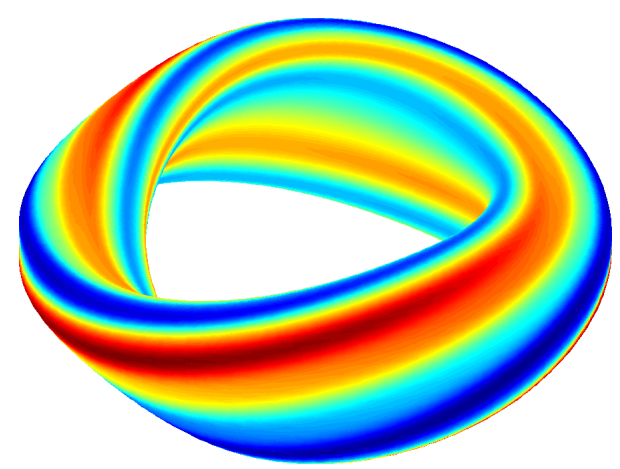

(b)

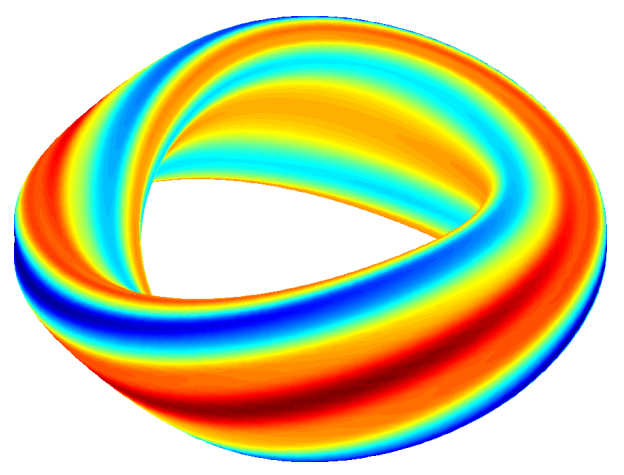

Fig. 5: Fourier spectra and mode structures of the radial velocity perturbations of the $n^{*}=2$ modes of the even mode family. Shown are the real parts of the eigenfunction Fourier spectra and the contour plots of (a) the sine-type, and (b) the cosine-type structure. In the upper plots the solid and dashed lines denote the contributions of the complex and conjugate-complex eigenfunctions, respectively. The largest contributions are marked by their poloidal and toroidal harmonics, $m / n$.
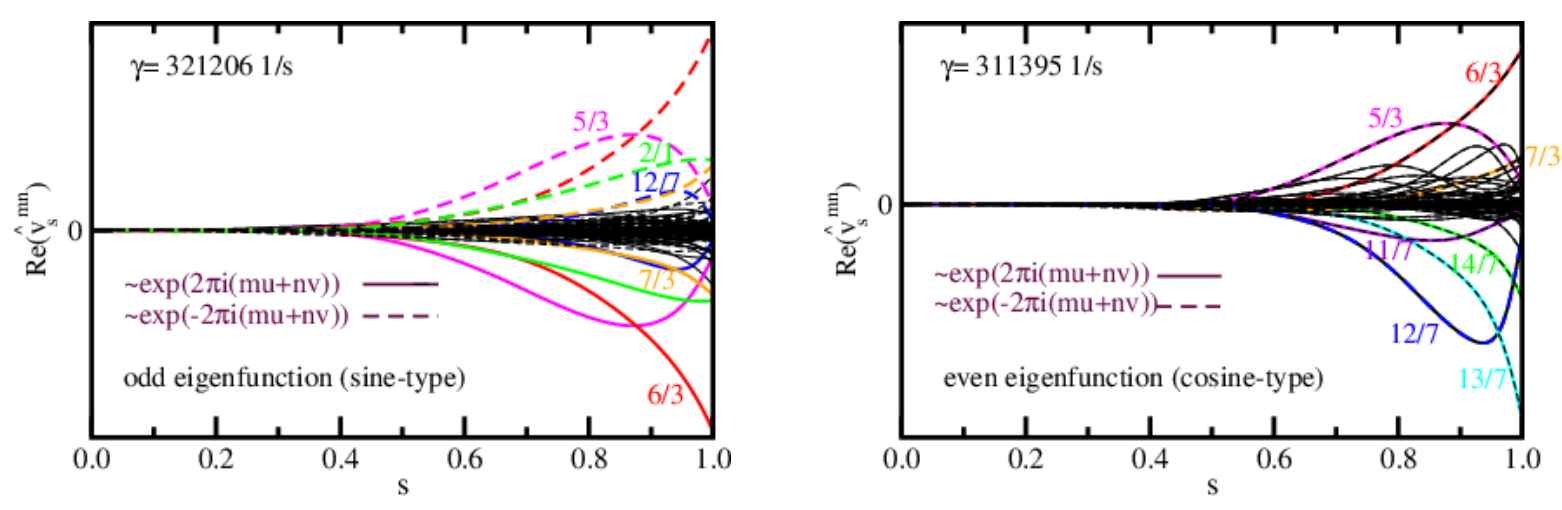
(a)

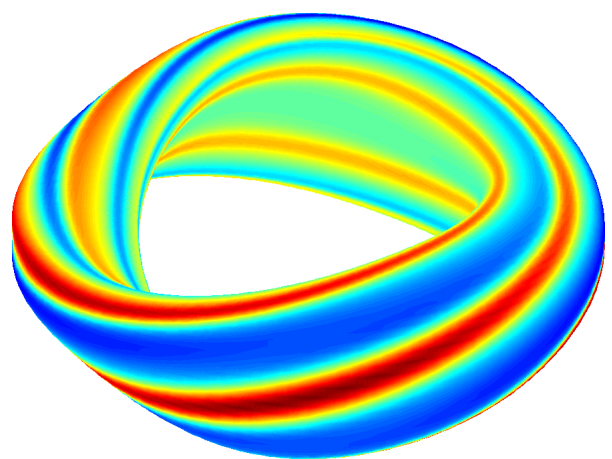

(b)

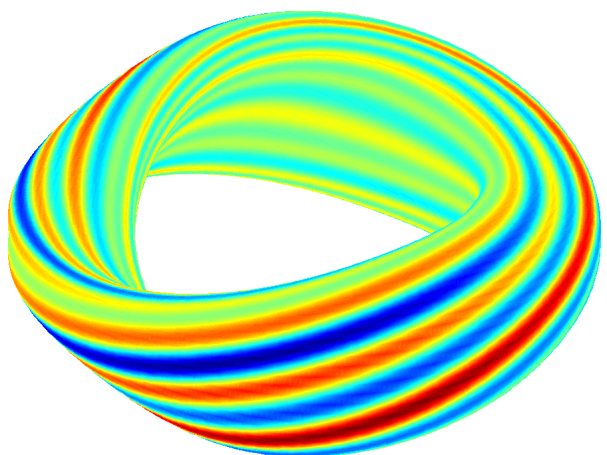

Fig. 6: Fourier spectra and modes structures of the radial velocity perturbations of the $n^{*}=3$ modes of the odd mode family.
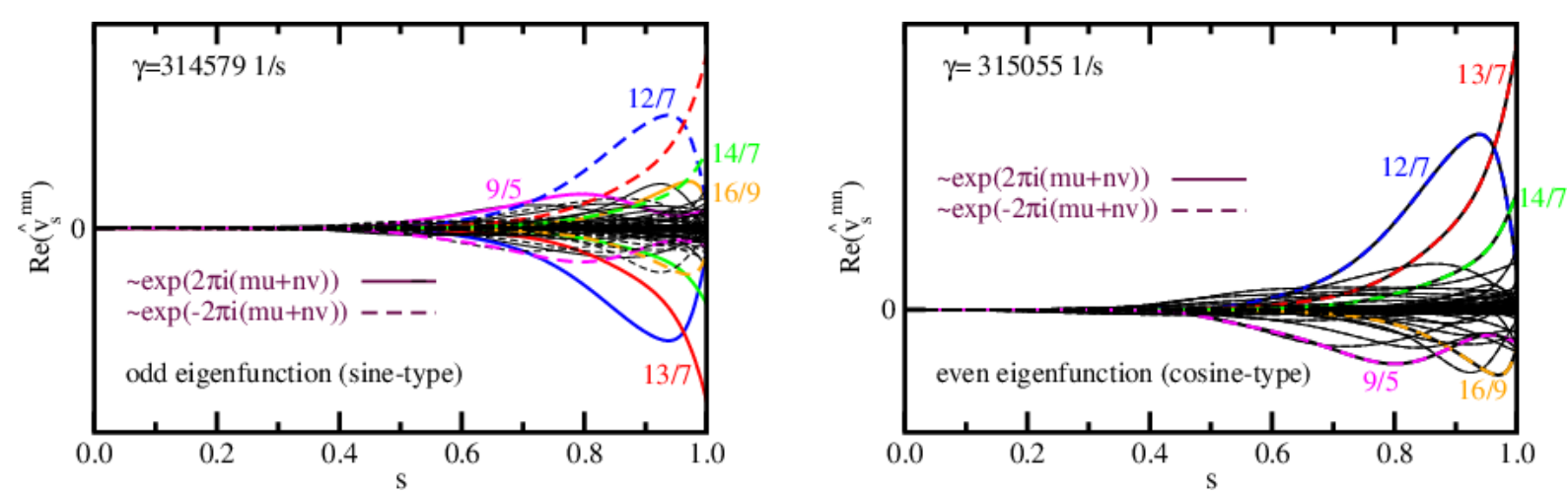

(a)

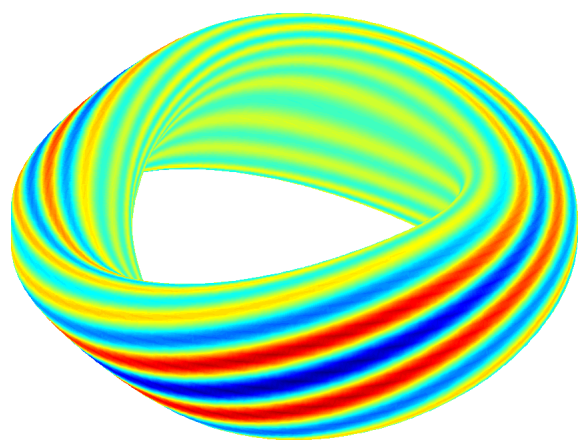

(b)

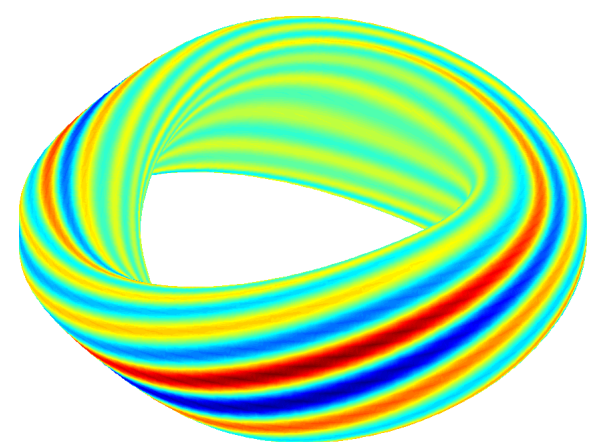

Fig. 7: Fourier spectra and mode structures of the radial velocity perturbations of the $n^{*}=7$ modes of the odd mode family.

As shown in Fig. 4, the eigenvalues obtained for the axisymmetric approximation are similar to the QA results. This similarity confirms the physical relevance of the chosen mode labeling. However, there is one important difference between the two equilibrium configurations. While the axisymmetric equilibrium is vertical unstable ( $n^{*}=0$ mode, see Figs 4 and 8 ), the QA equilibrium is stable with respect to this mode type due to its non-axisymmetric shape [26, 27]. Nevertheless, the $n=0$ harmonics contribute to the growth rates of the even mode family. As already described in detail in [17], the implemented many-valued current potentials [28] in the 
STARWALL part [3] of the CASTOR3D code allow a correct treatment of the $m=0, n=0$ harmonic. In order to allow for a net-poloidal current in the vacuum region that originates from this kind of perturbation, thin, ideally conducting, toroidal field coils are arranged in the vacuum region surrounding the plasma. The contribution of the $m=0, n=0$-harmonic to the growth rate of the vertical instability shown in Fig. 8 amounts to $\approx 10 \%$ (with $m=0: \gamma=306336$ 1/s, without $m=0: \gamma=2714401 / \mathrm{s}$ ). The Fourier spectra of the components of the velocity perturbation, shown in Fig. 8b-d, illustrate that the 0/0-harmonic contributes via the poloidal and toroidal velocity perturbations to the vertical instability. The effect of this harmonic on the growth rates of the even mode family of the QA equilibrium is much smaller. It amounts to $\approx 1 \%$ (with $m=0$ : $\gamma=338421 \mathrm{1} / \mathrm{s}$, without $m=0: \gamma=3347311 / \mathrm{s}$ ) for the $n^{*}=2$, sine-type mode, and is negligible small for all others.

Note, here and in the subsequent sections the characterization of the modes by sine-type or cosine-type always refers to the Fourier spectrum of the radial velocity perturbation. That is, the notations sine-type and cosine-type modes are the acronyms for modes with sine-type and cosine-type Fourier spectra of the radial velocity perturbations, respectively. The types of the eigenfunction Fourier spectra of all perturbed quantities are listed in Table 2. For the definitions of the radial velocity $\hat{v}_{s}^{m n}$, toroidal velocity $\hat{v}_{v}^{m n}$, poloidal velocity $\hat{v}_{u}^{m n}$, density $\hat{\rho}^{m n}$, temperature $\hat{T}^{m n}$, radial vector potential $\hat{A}_{s}^{m n}$, toroidal vector potential $\hat{A}_{v}^{m n}$, and poloidal vector potential Fourier harmonics $\hat{A}_{u}^{m n}$ see [17].

Table 2: Eigenfunction Fourier specta types of the perturbated quantities.

\begin{tabular}{ccccccccc}
\hline \hline mode type & $\hat{v}_{s}^{m n}$ & $\hat{v}_{v}^{m n}$ & $\hat{v}_{u}^{m n}$ & $\hat{\rho}^{m n}$ & $\hat{T}^{m n}$ & $\hat{A}_{s}^{m n}$ & $\hat{A}_{v}^{m n}$ & $\hat{A}_{u}^{m n}$ \\
\hline sine-type & sine & cosine & cosine & sine & sine & cosine & sine & sine \\
cosine-type & cosine & sine & sine & cosine & cosine & sine & cosine & cosine \\
\hline \hline
\end{tabular}

(a)
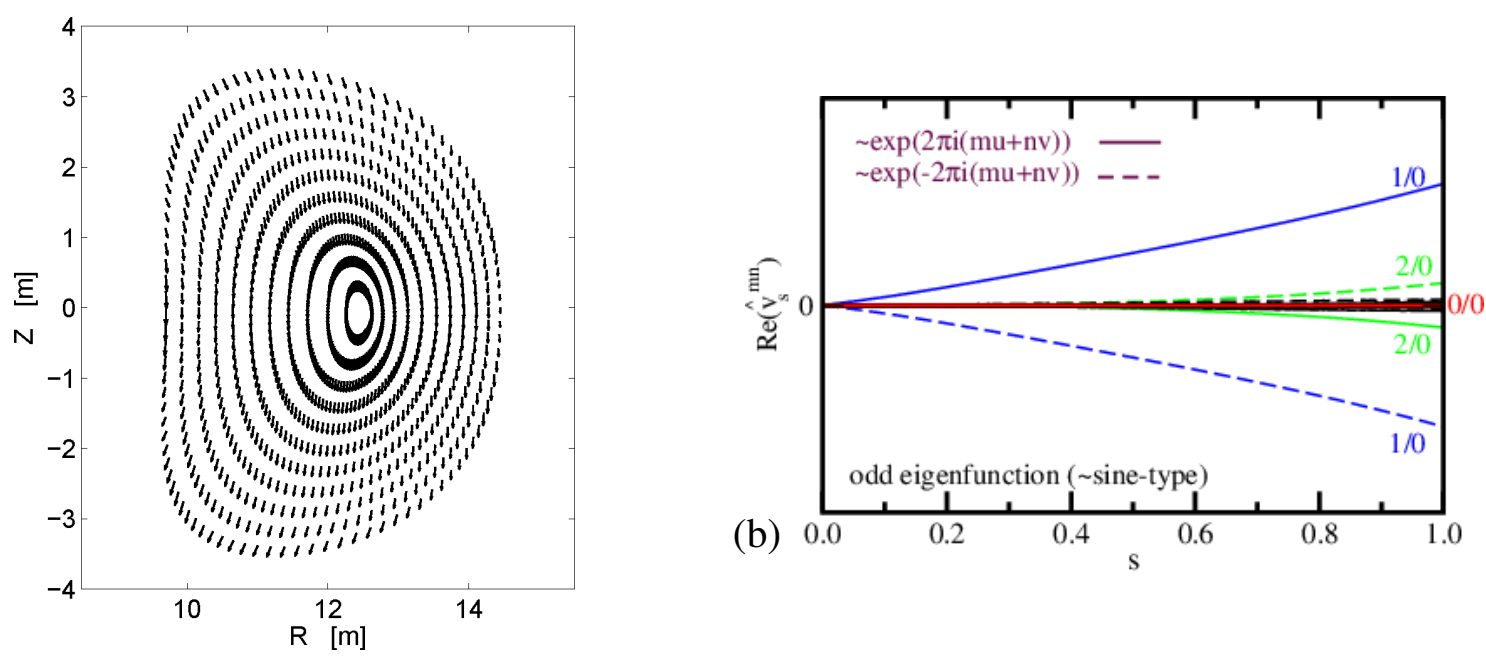

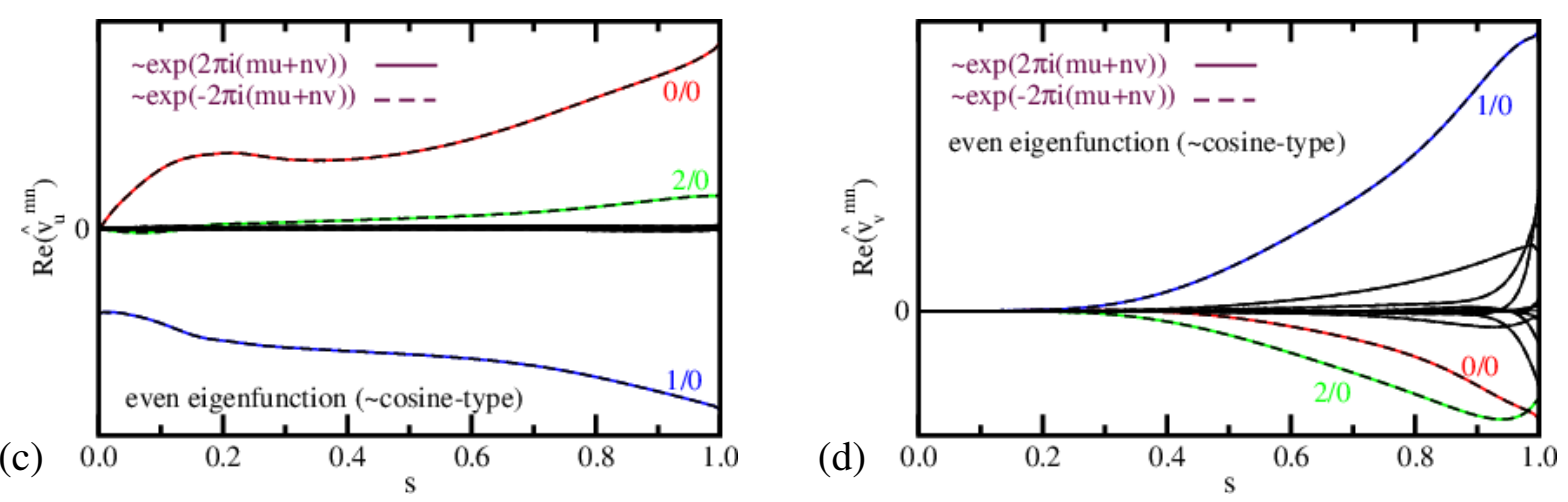

Fig. 8 (a) Mode structure of the vertical instability of the axisymmetric equilibrium. The vectors characterize the velocity perturbation in the $R-Z$ plane. (b)-(d) Fourier spectra of the radial, poloidal and toroidal velocity perturbations.

\subsection{Effect of the parallel viscosity}

For more realistic stability studies, especially in case of a rotating plasma, the viscous force has to be taken into account $[25,10,29,30]$. In a first step, we implemented the parallel viscous damping Eq.(6) in the CASTOR3D code. The dynamic viscosity coefficient, $\mu_{\|}$, may be a function of the radial coordinate, but here we use contstant values. Figure 9 shows the growth rates as functions of $\mu_{\|}$.
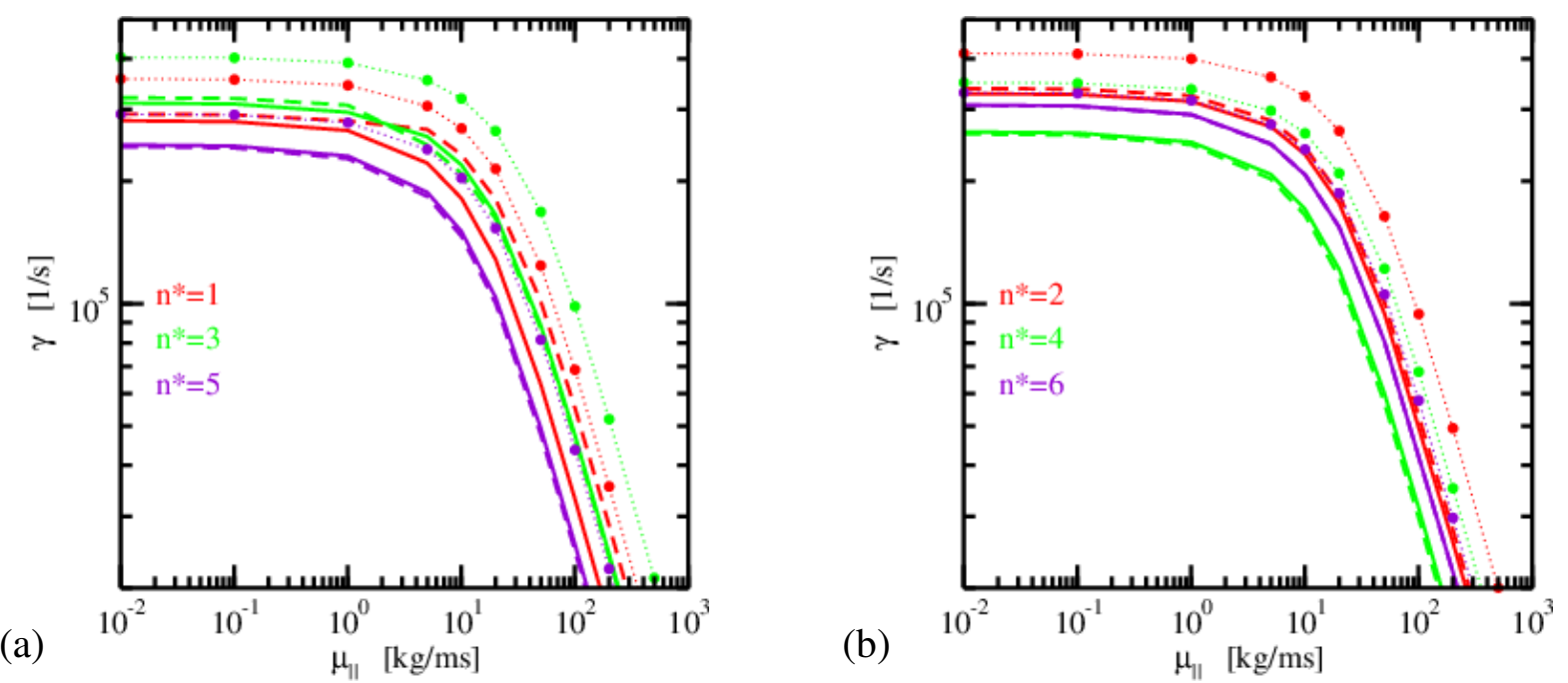

Fig. 9: Growth rates as functions of the parallel dynamic viscosity coefficient, $\mu_{\|}$, for various $n^{*}$-modes. (a) Odd mode family, and (b) even mode family of the QA equilibrium in comparison with the corresponding growth rates obtained for the axisymmetric approximation (dotted lines 
with full circles). The solid and dashed lines mark the cosine and sine-type modes of the QA configuration, respectively.

First of all, the growth rates of the QA equilibrium and of its axisymmetric approximation show the same behaviour. The parallel viscosity has a negligible effect on the growth rate for $\mu_{\|} \leq 1 \mathrm{~kg} / \mathrm{ms}$, while there is a slight damping for $1<\mu_{\|} \leq 10 \mathrm{~kg} / \mathrm{ms}$. Eventually, for $\mu_{\|}<10 \mathrm{~kg} / \mathrm{ms}$ the growth rates quickly decrease with growing parallel viscosity. A very rough estimate of $\mu_{\|} \approx \kappa_{\|} v_{i}^{t h} \rho_{a} / k_{\| \mid}$yields a value in the range of $1<\mu_{\|} \leq 10 \mathrm{~kg} / \mathrm{ms}$ for these high- $\beta$, collisionaless equilibria (see also Table 1) using $\kappa_{\|} \sim \sqrt{\pi}$, wave vector $k_{\|} \sim 1 / R_{0}$, density $\rho_{a} \sim 10^{-7} \frac{\mathrm{kg}}{\mathrm{m}^{3}}$, and ion thermal velocity $v_{i}^{t h} \sim 10^{6} \frac{\mathrm{m}}{\mathrm{s}}$. In the subsequent computations either no viscosity is taken into account, or $\mu_{\|}=50 \mathrm{~kg} / \mathrm{ms}$ is used. This large value has been chosen, because it leads to a noteworthy effect, and is, therefore, suitable to demonstrate the capability of the CASTOR3D code to deal with parallel viscosity in combination with plasma flow, and/or resistive wall structures in case of $2 \mathrm{D}$ and $3 \mathrm{D}$ equilibria.

As shown in Fig. 9a, the parallel viscosity damps the two $n^{*}=1$ modes of the QA equilibrium very differently. It reduces the growth rate of the sine-type mode less. Figures 10 and 11 show that the viscosity modifies the mode structure of the $n^{*}=1$, sine-type mode, while the structure of the cosine-type mode is almost unchanged.
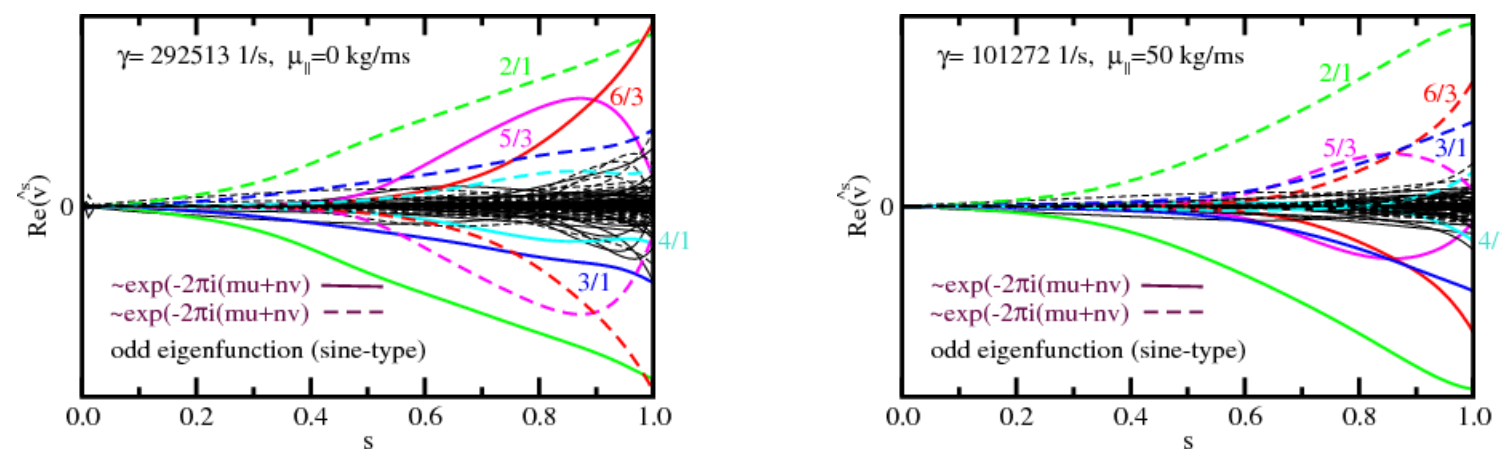

(a)

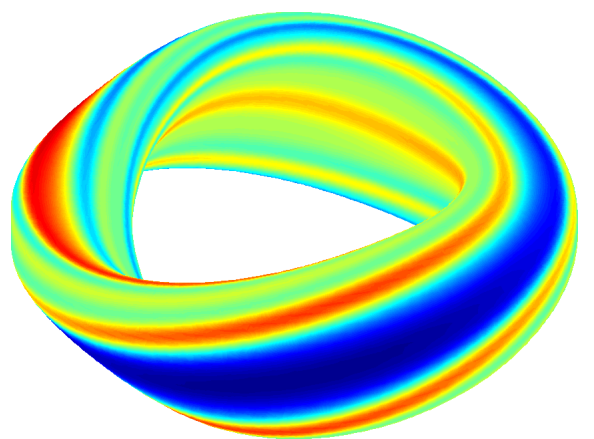

(b)

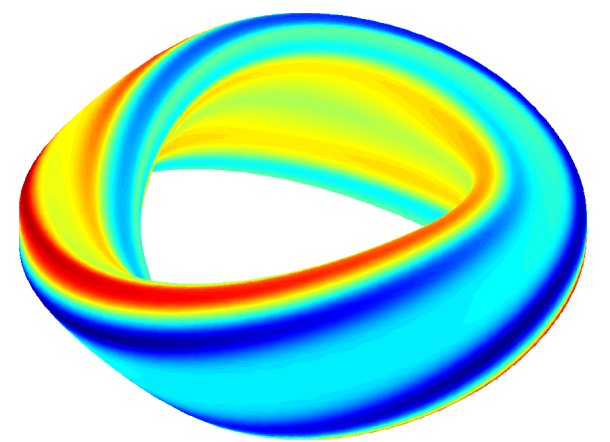

Fig. 10: Sine-type eigenfunctions: Fourier spectra and mode structures of the radial velocity perturbations of the $n^{*}=1$ mode of the odd mode family (a) without, and (b) with parallel viscosity. 

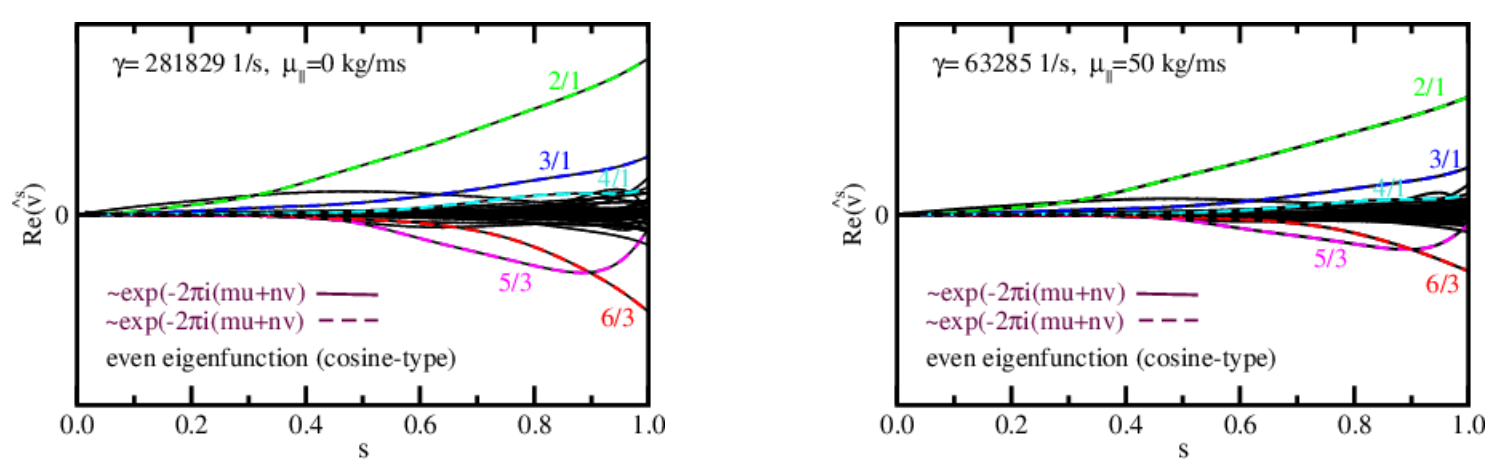

(a)

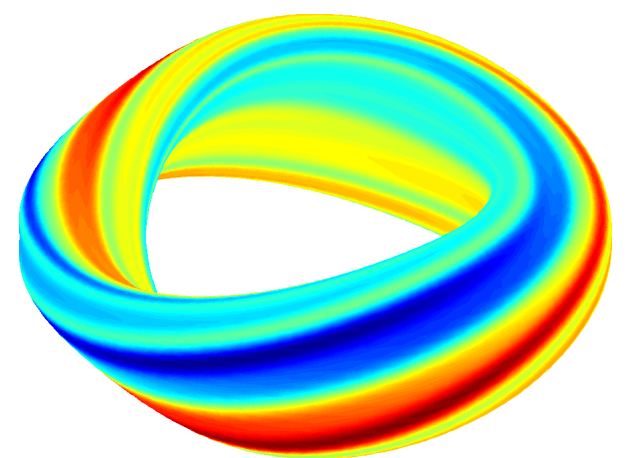

(b)

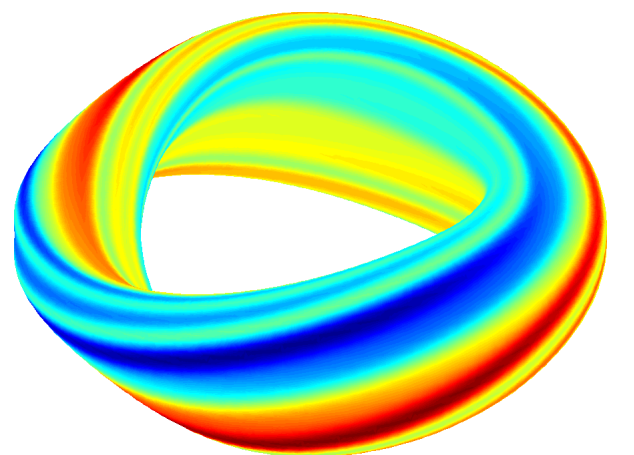

Fig. 11: Cosine-type eigenfunctions: Fourier spectra and mode structures of the radial velocity perturbations of the $n^{*}=1$ mode of the odd mode family (a) without, and (b) with parallel viscosity.

\subsection{Effect of the plasma flow}

Now we invesigate the effect of the plasma flow on the plasma stability of the QA equilibrium and its axisymmetric approximation. For this purpose, we use the normalized toroidal rotation profile shown in Fig. 12, which is defined by the polynomial $\Omega(s)=\Omega_{0}\left(1-0.7 s^{2}\right)$, with $\Omega_{0}$ being a free parameter that determines the plasma rotational frequency at the magnetic axis. We consider subsonic rotational frequencies in the range of $0 \leq \Omega_{0} \leq 100000 \mathrm{rad} / \mathrm{s}$, which correspond to Mach numbers $\leq 0.42$ or $\Omega_{0} / \Omega_{A} \leq 0.1$ with $\Omega_{A}$ being the Alfvén rotational frequency. Furthermore, we compare results obtained with $\left(\mu_{\|}=50 \mathrm{~kg} / \mathrm{ms}\right)$, and without parallel viscosity. Static equilibria are only approximations in case of large Mach numbers ( $\gtrsim 0.2[11]$ ). However, most of the presented results are obtained for smaller Mach numbers, and the used static equilibria are only test configurations to demonstrate the large variety of possible applications of the CASTOR3D code. 


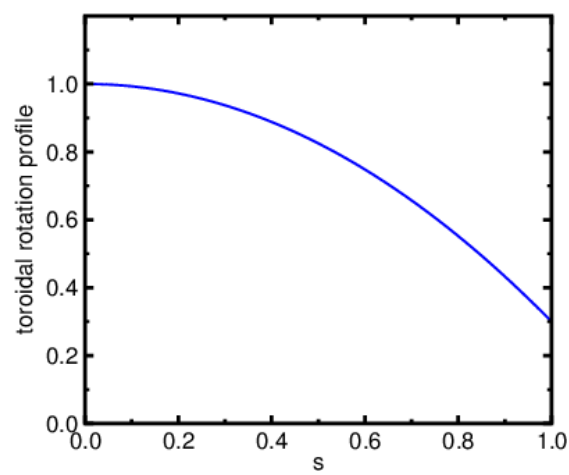

no viscosity

(a)

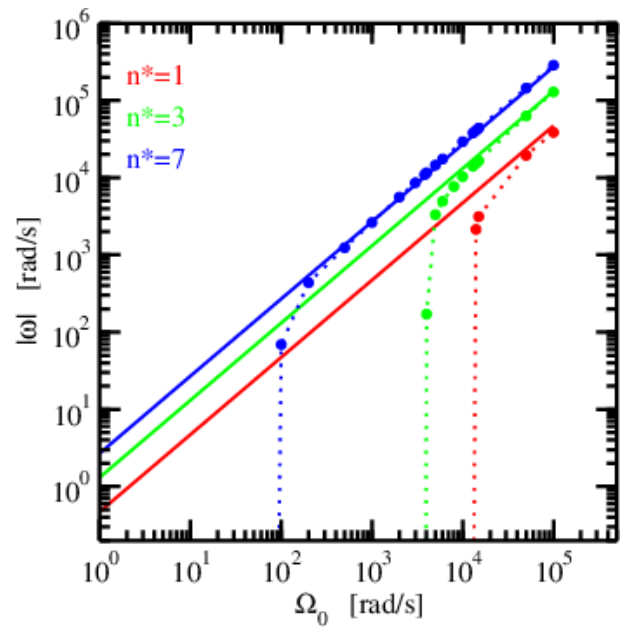

Fig. 12: Normalized torodial rotation profile.

$$
\mu_{\|}=50 \mathrm{~kg} / \mathrm{ms}
$$

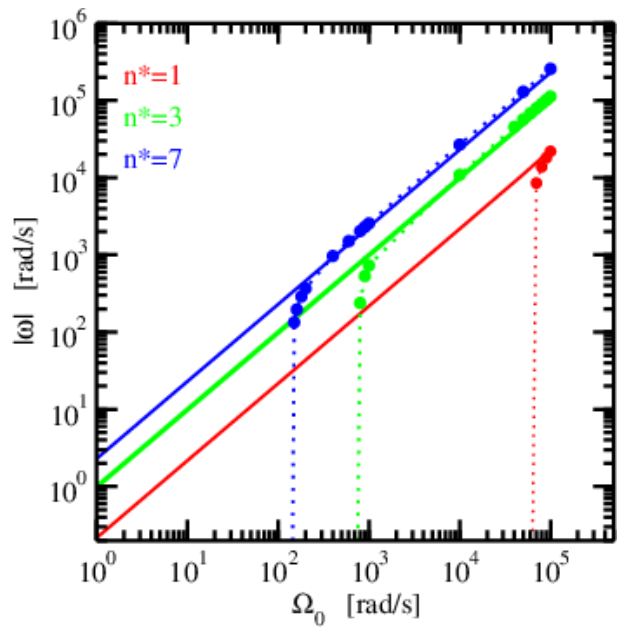

Fig. 13: Absolute values of the oscillation frequencies of the $n^{*}=1,3,7$ modes for the axisymmetric (solid lines) and the QA equilibrium (dotted lines with full circles) (a) without, and (b) with parallel ion viscosity $\left(\mu_{\|}=50 \mathrm{~kg} / \mathrm{ms}\right)$ taken into account. 
no viscosity
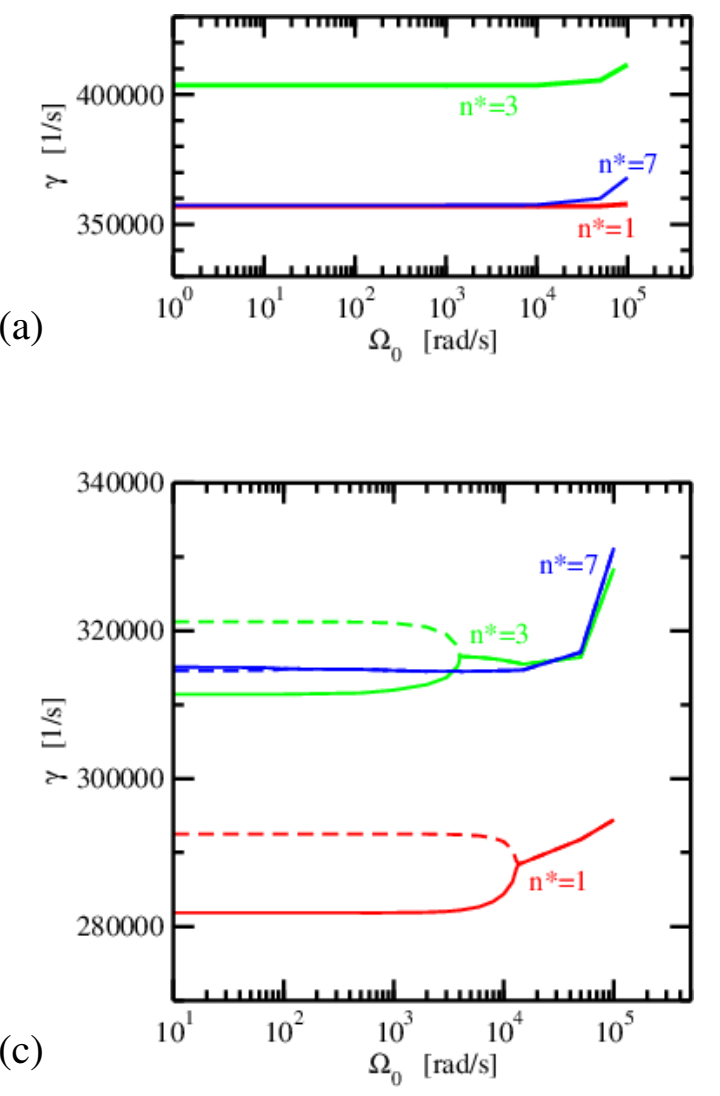

(e)

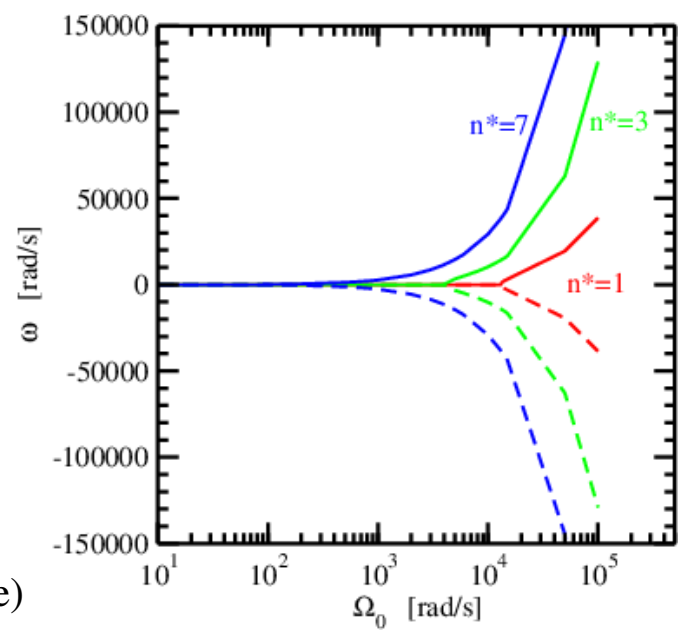

$$
\mu_{\|}=50 \mathrm{~kg} / \mathrm{ms}
$$
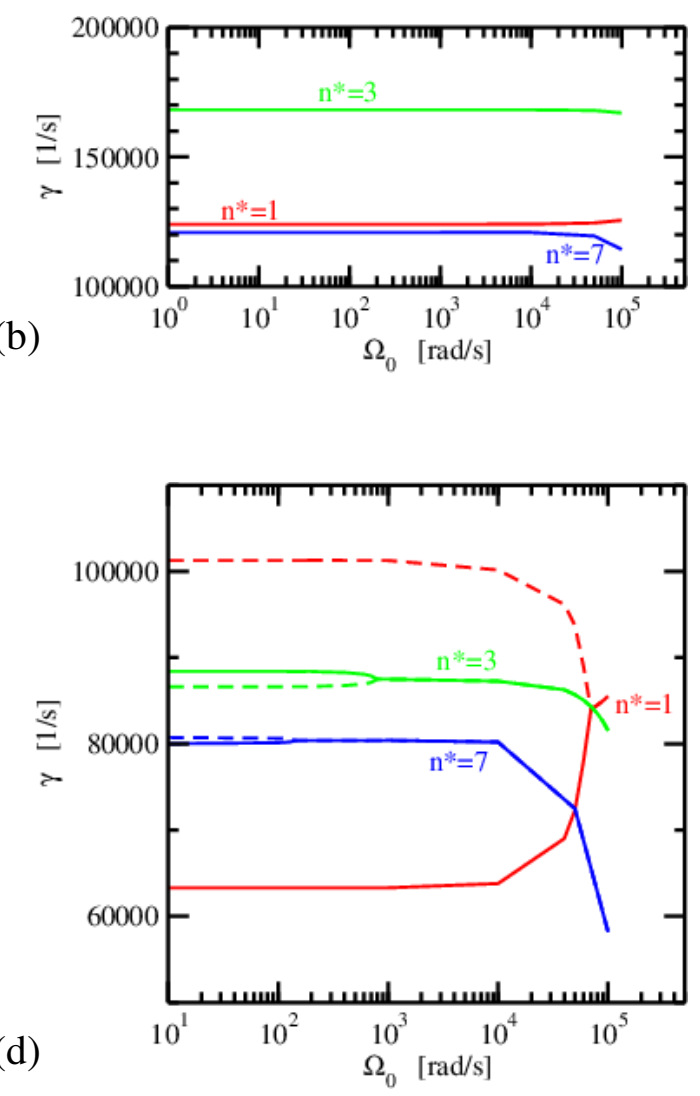

(f)

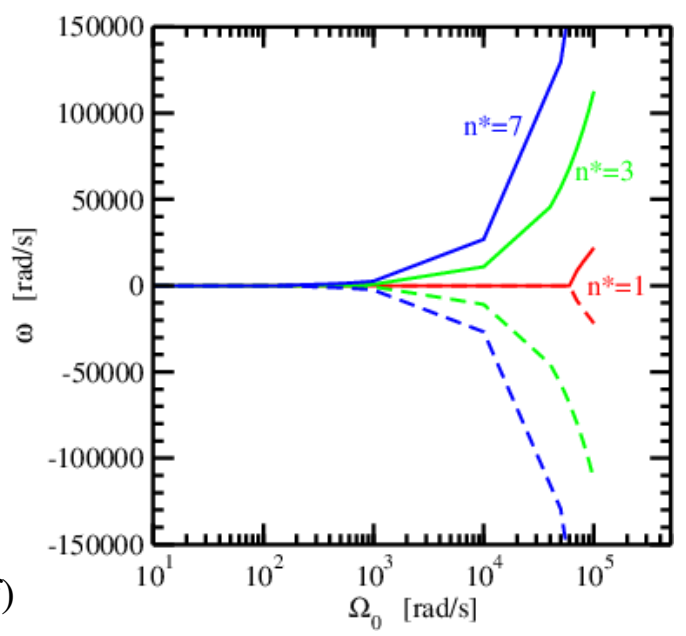

Fig. 14: (a-d) Growth rates and (e,f) oscillation frequencies as functions of $\Omega_{0}$ for the $n^{*}=1,3,7$ modes. (a,b) Growth rates of the axisymmetric equilibrium, as well as, (c,d) growth rates and $(e, f)$ oscillation frequencies of the QA equilibrium are obtained without (a,c,e) and with (b,d,f) parallel ion viscosity $\left(\mu_{\|}=50 \mathrm{~kg} / \mathrm{ms}\right)$ taken into account. The solid and dashed lines mark the two orthogonal solutions. 
Varying $\Omega_{0}$ in the defined range, we found a remarkable difference in the behaviour of the QA and the axisymmetric configurations. In the latter, the toroidal rotation of the plasma leads to an oscillation of the perturbations. The oscillation frequencies of the modes, $\omega$, are proportional to the rotation frequency of the plasma, $\Omega_{0}$, as shown in Fig. 13. In these double logarithmic representation $\omega$ (solid lines) linearly increases with $\Omega_{0}$. However, the behaviour of the QA configuration is different. There, a minimum plasma rotation frequency is required for the mode to oscillate. As illustrated in Fig. 13 (dotted lines with full circles), each mode type has its individual minimum frequency which we will call threshold frequency, $\Omega_{\text {thres }}$. While the mode doesn't oscillate for $\Omega_{0}<\Omega_{\text {thres }}$, the oscillation suddenly sets in for $\Omega_{0} \geq \Omega_{\text {thres }}$, quickly increases, and, finally, linearly grows with $\Omega_{0}$ as in the axisymmetric case. The reason for this behaviour is the following.

In axisymmetric geometry the two orthogonal solutions of the eigenvalue problem are degenerated, because the toroidal location of the mode doesn't play a role. This is different in 3D geometry. There, the two solutions belong to different mode structures which in general have different eigenvalues (see e.g. Fig. 5). However, in case of a rotating plasma, the difference of the two eigenvalues, $\Delta \gamma=\gamma_{1}-\gamma_{2}$, decreases with increasing $\Omega_{0}$, as shown in Figs 14c,d. When the two eigenvalues become equal, the mode starts to oscillate (see Figs 14e,f). Now, the various mode structures that the mode undergoes within an oscillation cycle have the same growth rate, and the mode is free to rotate.
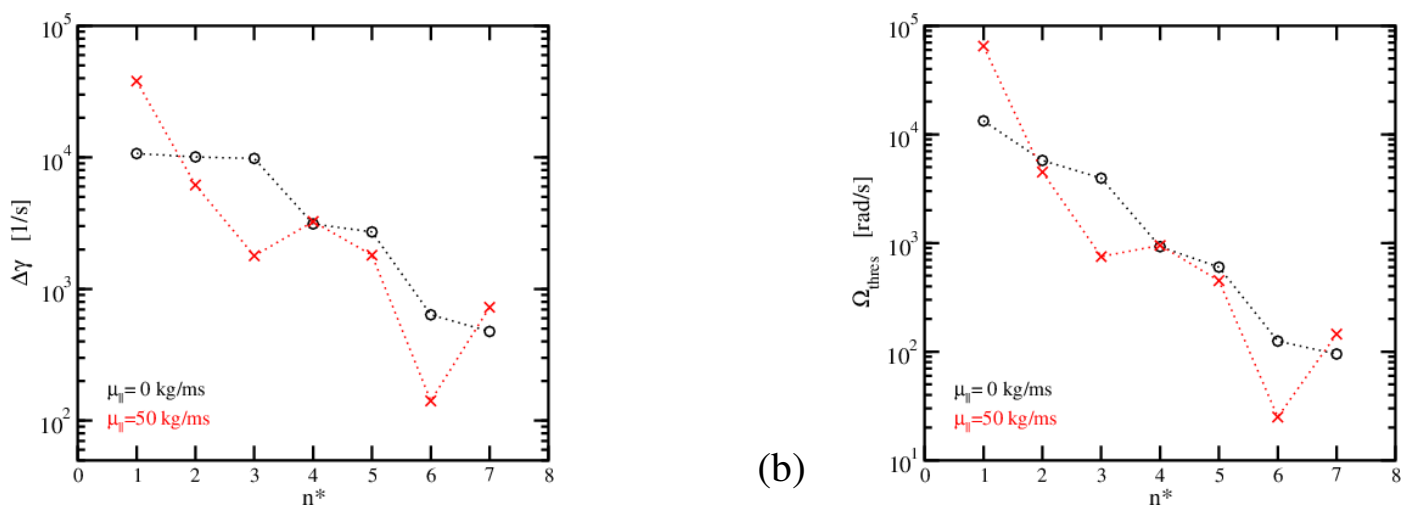

Fig. 15: (a) Differences of the non-degenerated growth rates of the non-rotating plasma, $\Delta \gamma=$ $\gamma_{1}-\gamma_{2}$, and (b) threshold frequencies, $\Omega_{\text {thres }}$, of the low- $n^{*}$ mode types of both mode families of the QA configuration. Black circles and red crosses mark the results obtained without, and with parallel viscosity taken into account.

The threshold frequency, $\Omega_{\text {thres }}$, is correlated with $\Delta \gamma$, as illustrated in Fig. 15. There, the differences of the non-degenerated eigenvalues of the non-rotating plasma, and $\Omega_{\text {thres }}$ are presented for the low- $n^{*}$ mode types of both mode families of the QA configuration. The two plots clearly show a correlation. The larger $\Delta \gamma$, the higher $\Omega_{\text {thres }}$. 

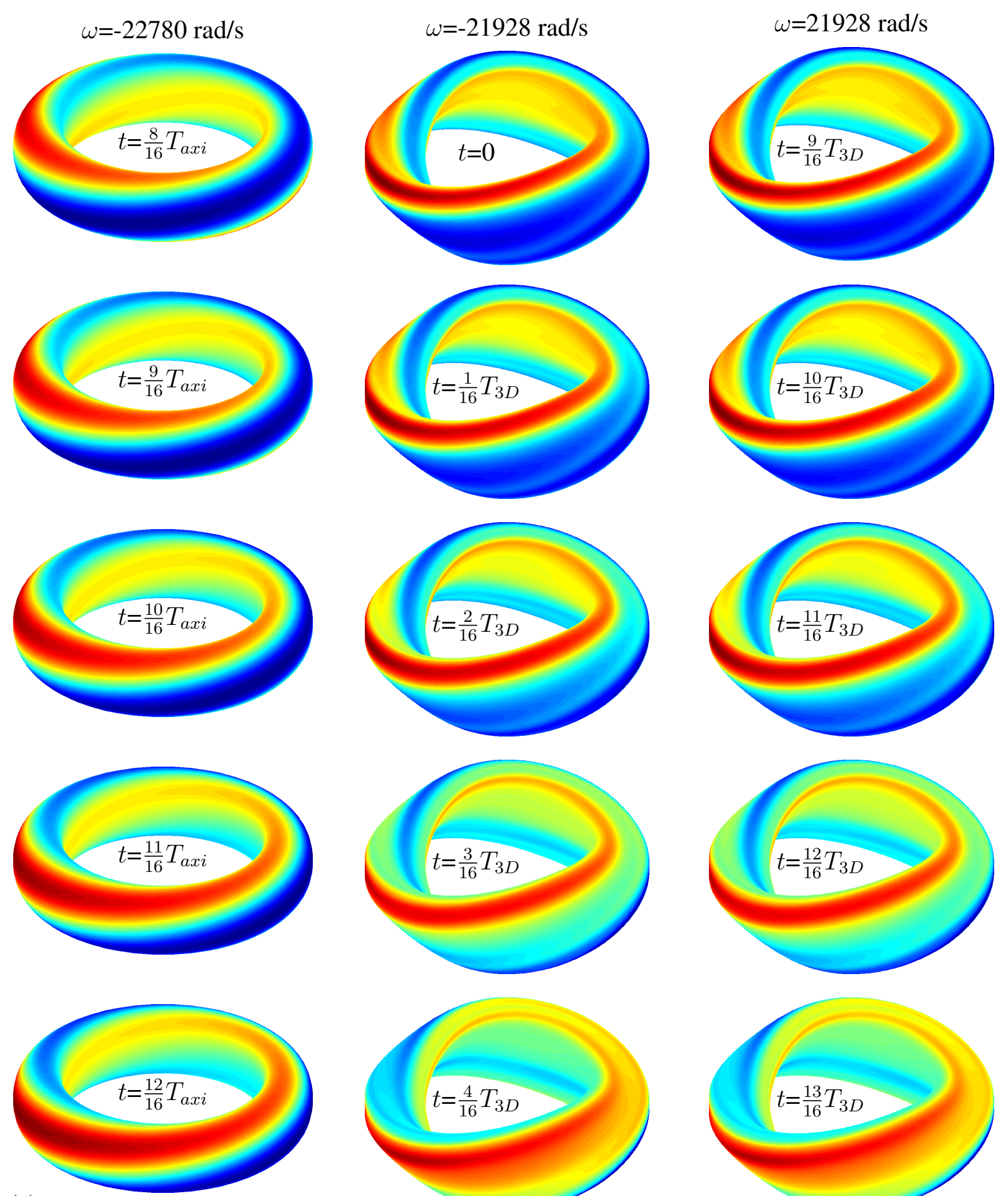

(a)
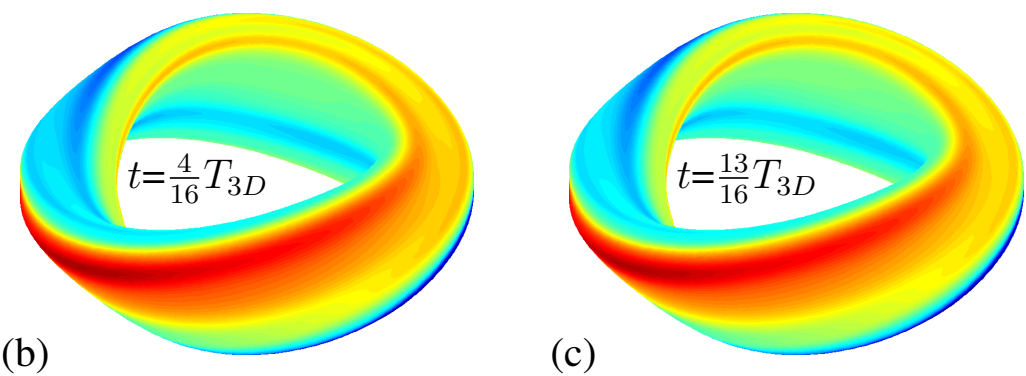

Fig. 16: Oscillation of the radial velocity perturbation of (a) the axisymmetric equilibrium , and the $Q A$ equilibrium with (b) $\omega<0$ and (c) $\omega>0$ for the $n^{*}=1$ mode type. Shown are various time steps within 1/4 oscillation cycle. 
In case of $\Omega_{0} \geq \Omega_{\text {thres }}$ ( $\Omega_{\text {thres }}=0$ for axisymmetric configurations), the solution of the eigenvalue problem yields two eigenvalues with $\gamma_{1}=\gamma_{2}$ and $\omega_{1}=-\omega_{2}$. The eigenvalues differ only in the signs of the oscillation frequencies. However, these opposite signs do not mean opposite rotation directions of the mode, but indicate a phase shift. That is, both modes undergo the same oscillation cycle, but the initial mode structures at $t=0$ are different. The phase shift is illustrated for the QA configuration in Fig. 16b,c. Additionally, a time sequence for the axisymmetric configuration is shown in Fig. 16a. The figures show the radial velocity perturbation of the $n^{*}=1$ mode type for equidistant time steps within $1 / 4$ oscillation cycle $T=\frac{2 \pi}{\omega} . T_{a x i}$ and $T_{3 D}$ denote the periods of one oscillation cycle of the axisymmetric and the QA configuration, respectively. The computations have been performed for $\Omega_{0}=1 \cdot 10^{5} \mathrm{rad} / \mathrm{s}$ and $\mu_{\|}=50 \mathrm{~kg} / \mathrm{ms}$. As indicated by the times, $t$, the phase shift of the QA configuration amounts to $\approx \frac{9}{16} T_{3 D}$. Starting the sequences for the QA cases $\omega<0$ (Fig. 16b) and $\omega>0$ (Fig. 16c) at $t=0$ and $t=\frac{9}{16} T_{3 D}$, respectively, the pattern are the same. In the axisymmetric case the mode oscillation simply corresponds to a toroidal rotation in direction of the plasma rotation (Fig. 16a), while in QA symmetry the oscillation looks more complicated (Fig. 16b,c).

\subsection{Effect of external wall structures}

External wall structures may be able to reduce the growth rates of external modes. While an ideal wall may stabilize the modes completely, a resistive wall only reduces the growth rates up to the resistive time scale of the wall. As already shown by Bondeson and Ward [25], external modes in tokamaks can be fully stabilized by resistive walls, if the plasma rotates at some fraction of the sound speed. Here, we demonstrate that all these findings also hold for 3D quasi-axisymmetric stellarators.
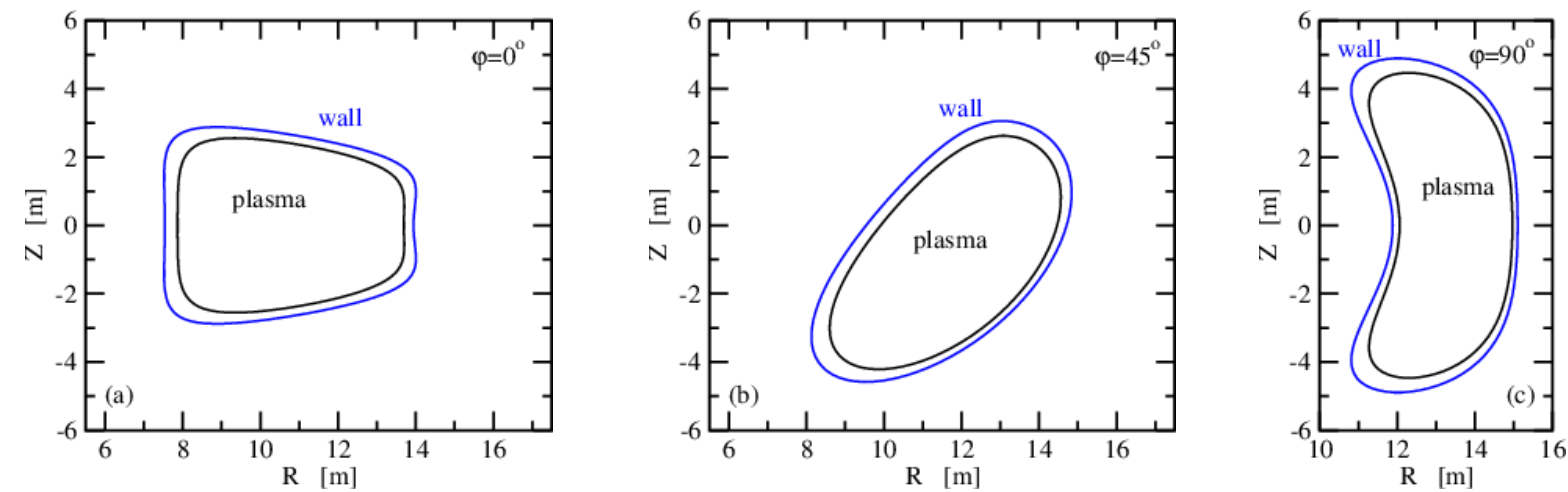

Fig. 17: Cross-sections of plasma boundary (black), and closed wall (blue) of the QA equilibrium at the toroidal angles $\varphi=0,45$ and $90^{\circ}$. The distance between plasma boundary and wall amounts to $\approx 25 \mathrm{~cm}$ at the outer midplane of the $\varphi=0^{\circ}$ cross-section. 
(a)

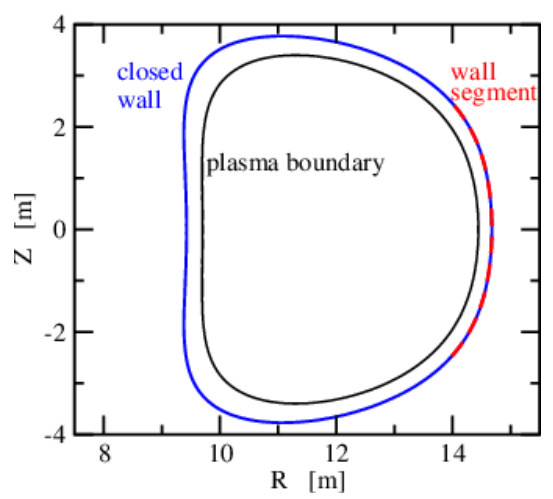

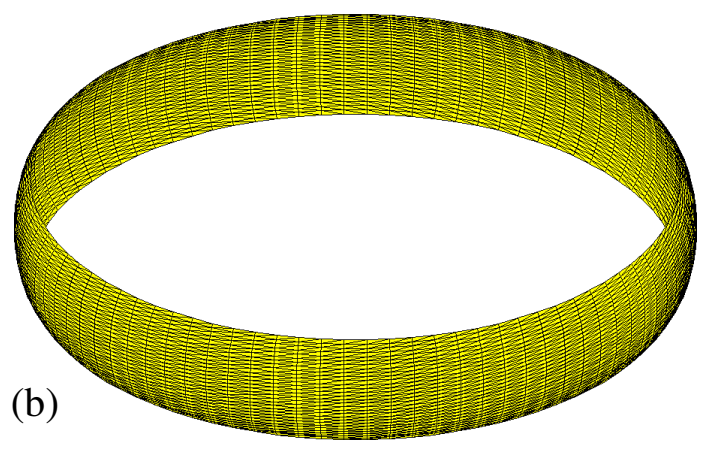

Fig. 18: (a) Cross-sections of plasma boundary (black), closed wall (blue), and wall segment (red) of the axisymmetric configuration. (b) $3 D$ representation of the axisymmetric wall segment. The distance between plasma boundary and wall amounts to $\approx 24 \mathrm{~cm}$ at the outer midplane.

Figures 17 and 18a show hypothetical closed walls for the QA and the axisymmetric equilibrium, respectively. The simple geometry of these walls is defined by 2D Fourier series. Furthermore, a wall segment composed by triangles is illustrated in Fig. 18b. The latter is used to study the growth rate of the vertical instability of the axisymmetric equilibrium as function of the wall resistivity, $\eta_{w}$, as shown in Fig. 19. Solving the extended eigenvalue problem for wall resistivities in the range of $10^{-9} \leq \eta_{w} \leq 1 \Omega \mathrm{m}$ (wall width: $\delta_{w}=0.5 \mathrm{~cm}$ ), the growth rates vary over 6 orders of magnitude from Resistive Wall Mode (RWM) time scales to the no wall limit. Here, the latter corresponds to $\gamma=2860861 / \mathrm{s}$.

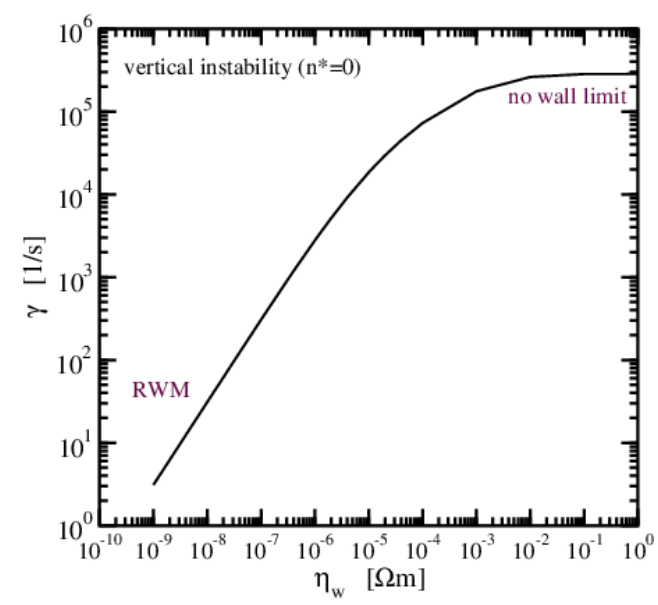

Fig. 19: Growth rate of the vertical instability as function of the resistivity, $\eta_{w}$, of the wall segment

We now study the dependencies of growth rates, $\gamma$, and oscillation frequencies, $\omega$, on the plasma-wall distances of the closed walls. As example, we choose the $n^{*}=2$ mode, which is the most unstable of the considered external kink modes (see Fig. 4). The wall distances, $d_{\text {wall }}$, are measured at the outer midplane of the $\varphi=0^{\circ}$ cross-sections of the axisymmetric and the QA equilibrium, respectively. The computations are performed for various cases: (i) 
ideal wall, no plasma rotation, (ii) resistive wall, no rotation, (iii) resistive wall, plasma rotation $\Omega_{0}=20000 \mathrm{rad} / \mathrm{s}$, and (iv) resistive wall, plasma rotation $\Omega_{0}=50000 \mathrm{rad} / \mathrm{s}$. A parallel ion viscosity of $\mu_{\|}=50 \mathrm{~kg} / \mathrm{ms}$ is taken into account. We use the same plasma rotation profile as in section 4.3 (see Fig. 12). Furthermore, for the resistive walls we assume a specific wall conductivity of $\sigma_{w}=56 \cdot 10^{6} \frac{1}{\Omega \mathrm{m}}$ (copper wall) and a wall width of $\delta_{w}=0.5 \mathrm{~cm}$ (within the thin wall approximation).

axisymmetric equilibrium

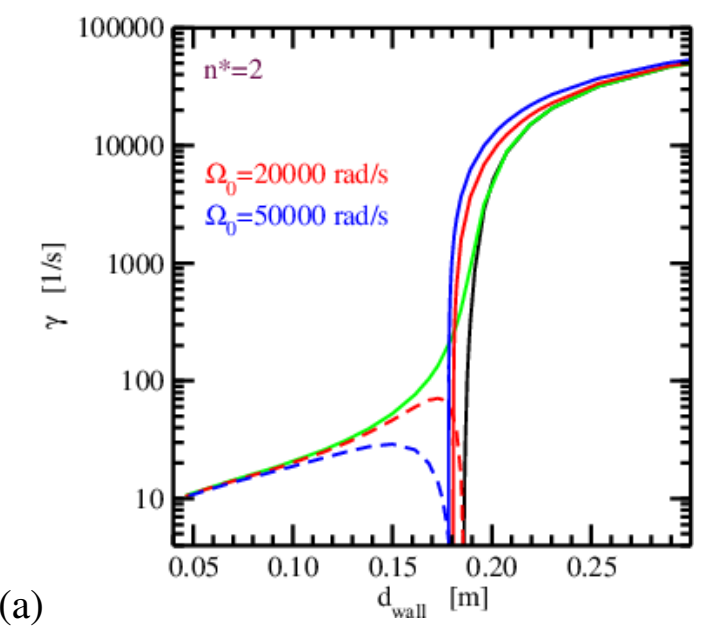

(b)

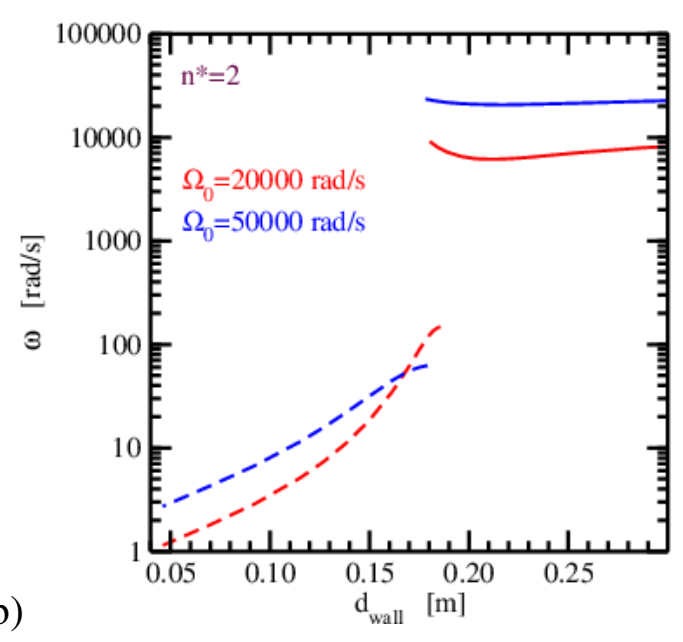

QA equilibrium
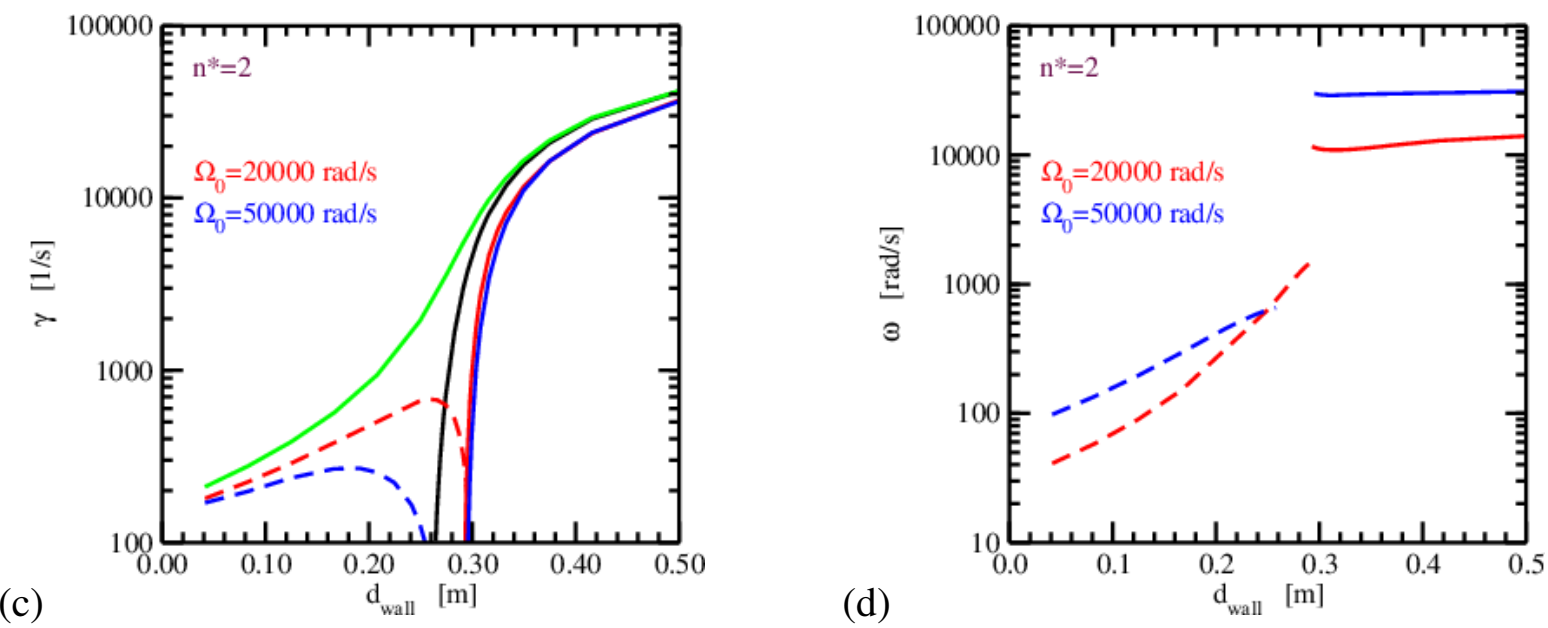

Fig. 20: (a,c) Growth rates, and (b,c) oscillation frequencies of the $n^{*}=2$ mode as functions of the wall distance, $d_{\text {wall }}$, for $(a, b)$ the axisymmetric and $(c, d)$ the $Q A$ equilibrium. The growth rates are obtained for an ideal closed wall (black), a resistive closed wall (green), and a resistive closed wall in combination with plasma flow (red: $\Omega_{0}=20000 \mathrm{rad} / \mathrm{s}$, blue: $\Omega_{0}=50000 \mathrm{rad} / \mathrm{s}$.) The solid red and blue curves mark the plasma modes, and the dashed curves the resistive wall modes. 
As shown in Fig. 20, the growth rates and oscillation frequencies obtained for the axisymmetric and the QA equilibrium show a similar behaviour. In both cases, the ideal wall stabilizes the $n^{*}=2$ mode at a certain distance, while the mode remains unstable for all distances in case of a resistive wall. Taking plasma rotation into account, two mode branches exist (as already described for tokamaks in [25]). (i) The growth rate of the so-called plasma mode decreases with shrinking wall distance, while it strongly oscillates. (ii) The growth rate of the resistive wall mode finally decreases with growing wall distance. The oscillation frequency of this mode is small. Furthermore, a narrow stability window arises in case of the QA equilibrium and a toroidal plasma rotation frequency of $\Omega_{0}=50000 \mathrm{rad} / \mathrm{s}$.

\section{Summary}

Additional extensions and improvements make the CASTOR3D code a versatilely applicable numerical tool. It computes growth rates and oscillation frequencies of MHD instabilities for 2D and 3D plasma equilibria, taking into account: (i) plasma resistivity, (ii) parallel viscosity, (iii) plasma flow in the direction of (quasi-)symmetry, and (iv) ideal and resistive wall structures. The coupling of the linear MHD and the vacuum equations via the perturbed magnetic vector potential allows the solution of an extended eigenvalue problem including all those physical effects. The MPI parallelization of the code, and the use of the parallelized SLEPc-Krylov-Schur solver for non-hermitian eigenvalue problems allow an efficient solution of large eigenvalue problems, which is essential in case of 3D configurations.

The linear stability properties of a quasi-axisymmetric stellarator equilibrium have been studied and compared with the results obtained for the axisymmetric approximation of this equilibrium. Both equilibria show similar properties with respect to growth rates of external kink modes, and damping of these modes due to parallel viscosity. Ideal walls may stabilize the external kink modes, while resistive walls can only reduce their growth rates down to resistive time scales. Depending on the plasma-wall distance of resistive wall structures, plasma modes and/or resistive wall modes are obtained in case of rotating axisymmetric and quasi-axisymmetric equilibria. Stability windows may exist. Besides these similarities, two differences in the stability properties of axisymmetric and quasi-axisymmetric equilibria have been found: (i) The QA equilibrium is vertical stable, while the axisymmetric equilibrium is vertical unstable. (ii) In case of a QA configuration a minimum plasma rotation frequency, $\Omega_{\text {thres }}$, is required for the mode to oscillate. Each mode type has its individual threshold frequency, which is correlated to the difference of the non-degenerated growth rates of the non-rotating plasma, $\Delta \gamma$. The larger 
$\Delta \gamma$, the higher $\Omega_{\text {thres }}$. In contrast to a QA configuration, the growth rates of the two orthogonal solutions of an axisymmetric configuration are degenerated, that is, $\Delta \gamma=0$ and $\Omega_{\text {thres }}=0$ for all mode types.

The reproduction of well known stability properties of axisymmetric equilibria, and the comparable results obtained for the QA equilibrium demonstrate that the CASTOR3D provides compelling results.

Further extensions of the CASTOR3D code are possible, e.g. the application of the code to equilibria with toroidal flow, or the implementation of heat conductivity, diamagnetic drift, etc.

\section{Acknowlegements}

The authors would like to thank Sophia Henneberg for providing the quasi-axisymmetric equilibrium, and Karl Lackner for several valuable discussions and suggestions.

This work has been carried out within the framework of the EUROfusion Consortium and has received funding from the Euratom research and training program 2014-2018 and 2019-2020 under grant agreement No 633053. The views and opinions expressed herein do not necessarily reflect those of the European Commision. 


\section{Appendix: Numerical accuracy}

The non-hermitian linear eigenvalue problem is solved by using the SLEPc Krylov-Schur Solver [19]. It provides the possibilities to compute all eigenvalues (the number of possible eigenvalues corresponds to the dimension of the matrix of the eigenvalue problem), or to determine a subgroup of eigenvalues the user is interested in. In the latter case initial guesses of eigenvalues and desired numbers of eigenvalues have to be provided. Since we are only interested in the most unstable low- $n$ external kink modes, we compute only sub-groups of eigenvalues. In a first step, it is necessary to determine $m$ - and $n$-harmonics that contribute to an eigenmode, that is, to determine all $m$ - and $n$-harmonics necessary for a converged eigenvalue solution.

In case of the axisymmetric approximation of the QA equilibrium each mode is defined by one toroidal harmonic $n$, and a spectrum of poloidal harmonics. Total number, $m_{\text {tot }}$, and region of the essential poloidal harmonics have to be determined numerically. Since we are interested in external kink modes, we use poloidal spectra centred around the smallest $m$-values that fulfill the condition $\frac{m}{n} \geq q_{b}$ for given $n$-numbers, and $q_{b}=1 / \iota_{b}=1.795$ being the safety factor at the plasma boundary (see also Table 1). Starting with these $m$ - harmonics and the correponding $n$ numbers, the number of poloidal harmonics is increased in each of the subsequent computations by two (e.g. computations for the $n=5$ external kink mode: 1 . computation: $m_{t o t}=1, m=9$, 2. computation: $m_{t o t}=3,8 \leq m \leq 10, \ldots, 9$. computation: $\left.m_{t o t}=17,1 \leq m \leq 17\right)$. In case of the $n=0$ vertical instability we start with $m=0,1$ and end up with $m=0,1, \ldots, 16$.

In Fig. 21 the growth rates as function of $m_{t o t}$ are shown for $n=0,1,2,5$ and 7 . These results demonstrate that 13 poloidal harmonics are enough to obtain converged eigenvalues with an inaccuracy $\leq 0.1 \%$ for this simple axisymmetric equilibrium. These results also show that the eigenvalues of the two orthogonal solutions per $n$ (represented by crosses and circles) are completely degenerated because of the axisymmetry of the equilibrium.

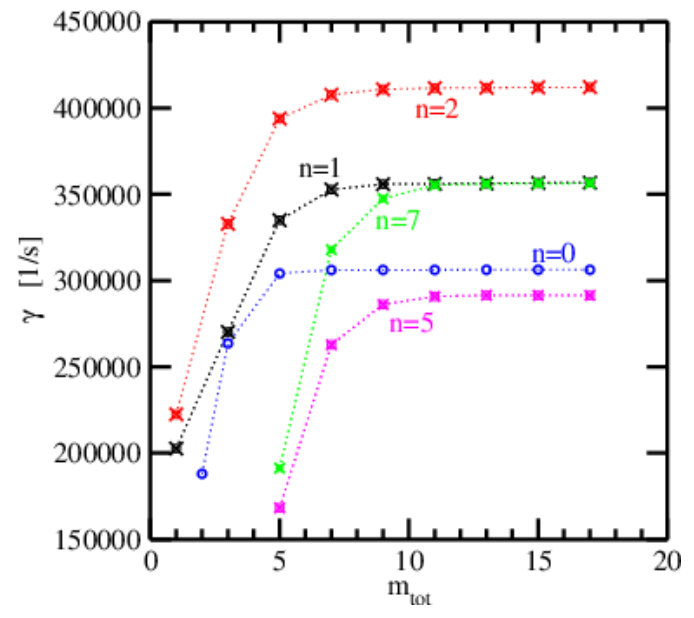

Fig. 21: Axisymmetric equilibrium: Growth rates of $n=0$ vertical instability, and $n=$ 1,2,5 and 7 external kink modes as functions of the total number of poloidal harmonics, $m_{\text {tot }}$. Crosses and circles mark the eigenvalues of the two orthogonal solutions of each nmode. 
The 3D geometry of the quasi-axisymmetric stellarator equilibrium causes a coupling of the toroidal-harmonics. That is, not only several $m$-harmonics, but also several $n$-harmonics contribute to an eigenmode. We have to determine the $m$ - and $n$-Fourier spectra of the essential harmonics contributing to a mode. This is done in an anlogue manner, as it is described above for the axisymmetric case, but now it is a $2 \mathrm{D}$ problem. Using the same poloidal Fourier spectra per $n$ as in the axisymmertic case, the total number of considered $n$-harmonics, $n_{\text {tot }}$, is increased step by step. Because of the two-periodic QA stellarator configuration, two mode families exist (odd mode family: $(n=1,3,5, \ldots)$, and even mode family: $(n=0,2,4, \ldots)$ ), which can be investigated separately. The toroidal harmonics of the two mode families don't couple because of the 2-periodic geometry [15].

(a)

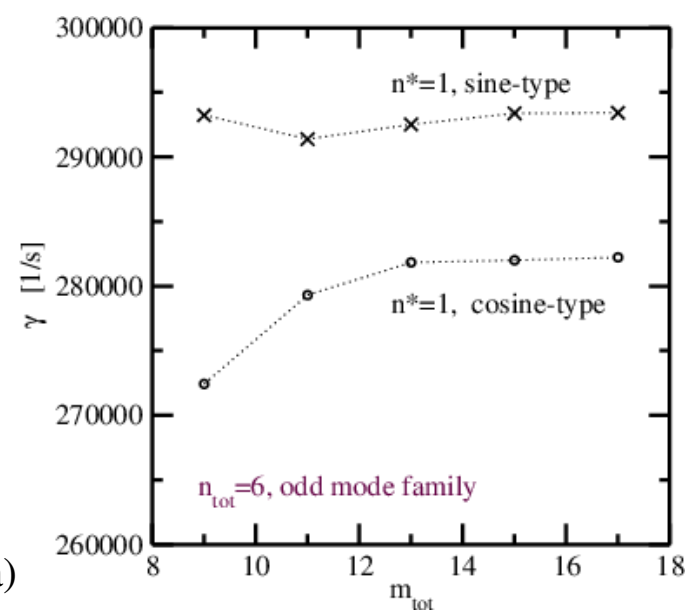

(c)

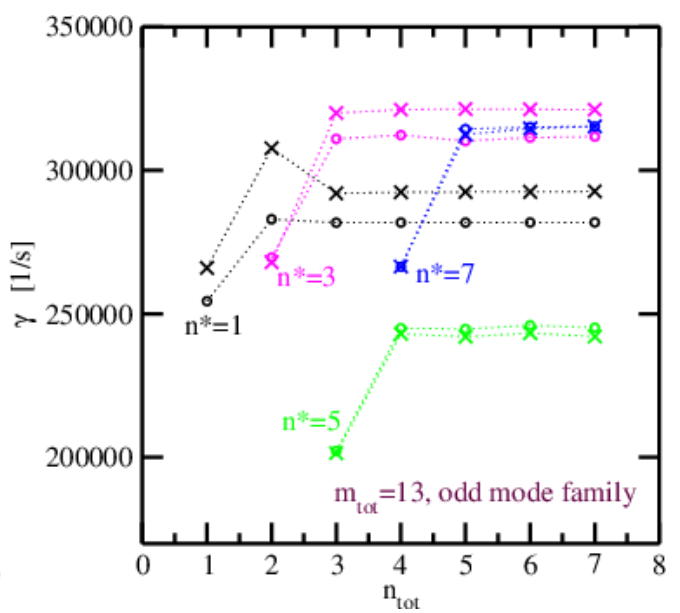

(b)

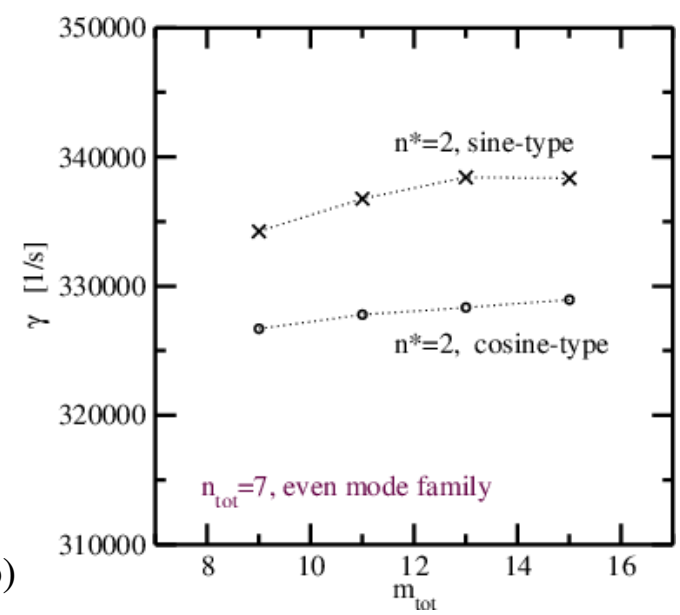

(d)

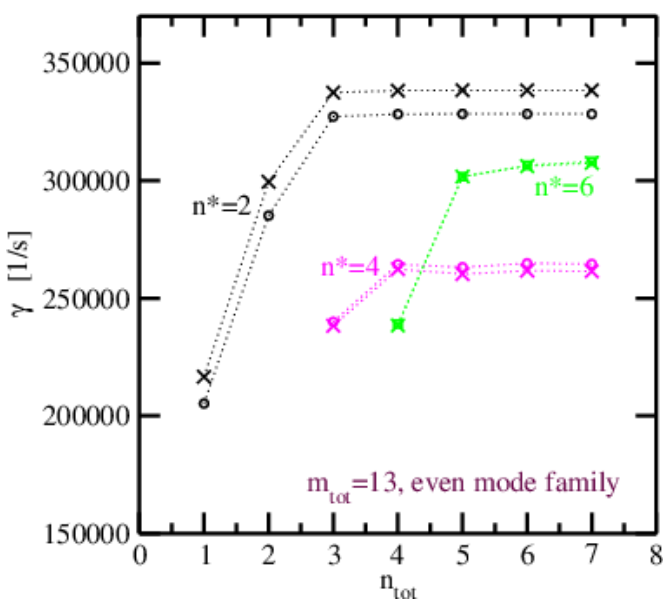

Fig. 22: Quasi-axisymmetric equilibrium: Growth rates of (a,c) the odd mode family, and (b,d) the even mode family of the QA equilibrium as functions of $(a, b) m_{t o t}$, and (c,d) $n_{t o t}$. The eigenvalues of the two orthogonal solutions of each $n^{*}$-mode are marked by crosses (sine-type Fourier spectrum of the radial velocity perturbation) and circles (cosine-type Fourier spectrum 
of the radial velocity perturbation).

Taking more than one toroidal harmonic into account, the solution of the eigenvalue problem yields eigenvalues of several unstable modes, which have to be identified. The $m$ - and $n$ harmonics of the largest Fourier harmonics are determined by analyzing the eigenfunctions of the radial velocity perturbation (see sect. 4.1, Figs 5-7, upper plots). Usually, the Fourier spectra are clearly dominated by one toroidal harmonic. We use this $n$-value to characterize the mode. Throughout the paper, the eigenvalues and the corresponding modes are labeled by this $n$-value, which we call $n^{*}$, in order to avoid confusion with the toroidal harmonic, $n$. In case of the modes of the axisymmetric equilibrium, it is $n^{*}=n$. The knowledge of the eigenvalue spectra (Fig. 22), and the already identified eigenfunction Fourier spectra help to characterize ambigous mode structures (see also sect. 4.1).

In order to make sure that the most unstable, low- $n^{*}$ external kink modes $\left(n^{*} \leq 7\right)$ have been found, a scan over initial guesses of the eigenvalues has been performed. No solutions with larger eigenvalues could be found. All results presented in this publication have been obtained by solving the linear eigenvalue problems for the Fourier ensembles listed in Table 3a,b. Figures 22a-d clearly show that these harmonics (which correspond to $m_{t o t}=13$ per $n$, and $n_{t o t}=6$ (odd mode family) and $n_{t o t}=7$ (even mode family), respectively) are sufficient to determine the eigenvalues with an inaccuracy $\lesssim 1 \%$.

\begin{tabular}{cc} 
Table 3a: odd mode family \\
\hline \hline$n$ & $m$-interval \\
\hline 1 & $-4 \leq m \leq 8$ \\
3 & $0 \leq m \leq 12$ \\
5 & $3 \leq m \leq 15$ \\
7 & $6 \leq m \leq 10$ \\
9 & $10 \leq m \leq 22$ \\
11 & $15 \leq m \leq 27$ \\
- & - \\
\hline \hline
\end{tabular}

\begin{tabular}{cc}
\multicolumn{2}{c}{ Table 3b: even mode family } \\
\hline \hline$n$ & $m$-interval \\
\hline 0 & $0 \leq m \leq 12$ \\
2 & $-2 \leq m \leq 10$ \\
4 & $1 \leq m \leq 13$ \\
6 & $4 \leq m \leq 16$ \\
8 & $8 \leq m \leq 20$ \\
10 & $11 \leq m \leq 23$ \\
12 & $15 \leq m \leq 27$ \\
\hline \hline
\end{tabular}

\section{References}

[1] STRUMBERGER, E., MERKEL, P., SEMPF, M., and GÜNTER, S., Phys. Plasmas 15 (2008) 056110. 
[2] VILLONE, F., LIU, Y. Q., PACCAGNELLA, R., BOLZONELLA, T., and RUBINACCI, G., Phys. Rev. Letters 100 (2008) 255005.

[3] MERKEL, P. and STRUMBERGER, E., Linear MHD stability studies with the STARWALL code, http://arxiv.org/abs/1508.04911, 2015.

[4] WILLENSDORFER, M., STRUMBERGER, E., SUTTROP, W., et al., Nuclear Fusion 57 (2017) 116047.

[5] EVANS, T. E., FENSTERMACHER, M. E., MOYER, R. A., et al., Nuclear Fusion 48 (2008) 024002.

[6] SUTTROP, W., FUCHS, J. C., FISCHER, R., et al., Fusion Eng. Des. 88 (2013) 446.

[7] NÜHRENBERG, J., LOTZ, W., and GORI, S., Quasi-axisymmetric tokamaks, in Theory of Fusion Plasmas, ISPP-15, p. 3, Varenna, Italy, 1994, Proc. Joint Varenna-Lausanne Int. Workshop.

[8] BOOZER, A. H., Phys. Fluids 25 (1982) 520.

[9] HENNEBERG, S. A., DREVLAK, M., NÜHRENBERG, C., et al., Nuclear Fusion 59 (2019) 026014.

[10] CHU, M. S., GREENE, J. M., JENSEN, T. H., MILlER, R. L., and BONDESON, A., Phys. Plasmas 2 (1995) 2236.

[11] STRUMBERGER, E., GÜNTER, S., MERKEL, P., RIONDATO, S., and SCHWARZ, E., Nuclear Fusion 45 (2005) 1156.

[12] CHAPMAN, I. T., SHARAPOV, S. E., HUYSMANS, G. T. A., and MIKHAILOVSKII, A. B., Phys. Plasmas 13 (2006) 062511.

[13] LIU, Q., CHU, M. S., CHAPMAN, I. T., and HENDER, T. C., Phys. Plasmas 15 (2008) 112503.

[14] VILLONE, F., LUI, Y., RUBINACCI, G., and VENTRE, S., Nuclear Fusion 50 (2010) 125011 .

[15] NÜHRENBERG, C., Phys. Plasmas 3 (1996) 2401.

[16] ANDERSON, D. V., COOPER, W. A., GRUBER, R., MERAZZI, S., and SCHWENN, U., Int. J. Supercomput. Appl. 4 (1990) 34. 
[17] STRUMBERGER, E. and GÜNTER, S., Nuclear Fusion 57 (2017) 016032.

[18] STRUMBERGER, E., MERKEL, P., TICHMANN, C., and GÜNTER, S., Linear stability studies in the presence of $3 \mathrm{~d}$ wall structures, in 38th EPS Conf. on Plasma Phys., ECA Vol. 35G P5.082, Strasbourg, France, 2011.

[19] ROMIN, J. E., CAMPOS, C., ROMERO, E., and TOMÁS, A., SLEPc Users Manual, Technical report dsic-ii/24/02, Universidad Politecnica de Valencia, http://slepc.upv.es/documentation/slepc.pdf, 2018.

[20] KERNER, W., GOEDBlOED, J. P., HUYSMANS, G. T. A., POEDTS, S., and SCHWARZ, E., J. Comput. Phys. 142 (1998) 271.

[21] HELANDER, P. and SIMAKOV, A. N., Phys. Rev. Lettters 101 (2008) 145003.

[22] HUYSMANS, G. T. A., GOEDBLOED, J. P., and KERNER, W., Phys. Fluids B 5 (1993) 1545.

[23] HIRSHMAN, S. P. and WHITSON, J. C., Phys. Fluids 26 (1983) 3553.

[24] HIRSHMAN, S. P., VAN RIJ, W. I., and MERKEL, P., Comput. Phys. Commun. 43 (1986) 143.

[25] BONDESON, A. and WARD, D. J., Phys. Rev. Lett. 72 (1994) 2709.

[26] REDI, M. H., NÜHRENBERG, C., COOPER, W. A., et al., Vertical and kink mode stability calculations for current carrying quasiaxial stellarators, in 26th EPS Conf. on Plasma Phys., ECA Vol. 23J P4.085, Maastricht, The Netherlands, 1999.

[27] REIMAN, A., FU, G., HIRSHMAN, S., et al., Plasma Phys. Control. Fusion 41 (1999) B273.

[28] LÜST, R. and MARTENSEN, E., Z. Naturforschung 15a (1960) 706.

[29] GREGORATTO, D., BOndESON, A., CHU, M. S., and GAROFAlO, A. M., Plasma Phys. Control. Fusion 43 (2001) 1425.

[30] LIU, C. and LIU, Y., J. Plasma Phys. 81 (2015) 905810516. 